\title{
Genome-wide Identification and Expression Analysis of NAC Transcription Factor Family Genes during Fruit and Kernel Development in Siberian Apricot
}

\author{
Wanyu Xu \\ State Key Laboratory of Tree Genetics and Breeding, Non-timber Forest Research and \\ Development Center, Chinese Academy of Forestry, Zhengzhou 450003, China; Key Laboratory \\ of Non-timber Forest Germplasm Enhancement and Utilization of National Forestry and \\ Grassland Administration, Zhengzhou 450003, China; and Nanjing Forestry University, Nanjing \\ 210037, China

\begin{abstract}
Chen Chen, Ningning Gou, Mengzhen Huang, Tana Wuyun, Gaopu Zhu, Han Zhao, Huimin Liu, and Lin Wang

State Key Laboratory of Tree Genetics and Breeding, Non-timber Forest Research and Development Center, Chinese Academy of Forestry, Zhengzhou 450003, China; and Key Laboratory of Non-timber Forest Germplasm Enhancement and Utilization of National Forestry and Grassland Administration, Zhengzhou 450003, China
\end{abstract}

\begin{abstract}
AdDitional INDEX wORDs. duplication events, phylogeny construction, Prunus sibirica, Rosaceae, synteny
Abstract. The NAC (NAM, ATAF1/2, and CUC2) family is a group of plant-specific transcription factors that have vital roles in the growth and development of plants, and especially in fruit and kernel development. This study aimed to identify members of the NAC gene (PSNACs) family and investigate their functions in siberian apricot (Prunus sibirica). A total of 102 predicted PsNAC proteins (PsNACs) were divided into 14 clades and the genes were mapped to the eight chromosomes in siberian apricot. The PsNACs of the same clade had similar structures. A synteny analysis showed that the PsNACs had close relationships with the NAC genes of japanese apricot (Prunus mume). An expression pattern analysis of the PsNACs revealed many differences in various tissues and at different stages of fruit and kernel development. All eight PsNACs in clade XI have crucial roles in fruit and kernel development. Seven PsNACs (PsNACs 18, 64, 23, 33, 9, 4, and 50) in clades I, III, VI, VII, and XIII are related to fruit development. Eight PsNACs (PSNACs 6, 13, 46, 51, 41, 67, 37, and 59) in clades I, II, V, VIII, and XIII are involved in fruit ripening. Five PsNACs (PsNACs 6, 94, 41, 32, and 17) in clades I, IV, V, VII, and XI regulated the rapid growth of the kernel. Four PsNACs (PsNACs 50, 4, 67, and 84) in clades I, III, V, and XIII affected the hardening of the kernel. Four PsNACs (PsNACs 17, 82, 13, and 51) in clades II, XI, and IX acted on kernel maturation. We have characterized the NAC genes in siberian apricot during this study. Our results will provide resources for future research of the biological roles of PsNACs in fruit and kernel development in siberian apricot.
\end{abstract}

Siberian apricot (Prunus sibirica) belongs to the family Rosaceae and is widely distributed in northern and northeastern China, eastern and southeastern Mongolia, eastern Siberia, and the Maritime Territory of Russia (Wang and Yu, 2012). Siberian apricot can grow in harsh environmental conditions and has been used for afforestation in northern China because of its strong environmental adaptability (Wang et al., 2017; Zhang et al., 2006). The kernels of siberian apricot have commercial value; for example, they can be processed into protein drinks

Received for publication 9 Oct. 2020. Accepted for publication 23 Mar. 2021. Published online 2 June 2021.

This work was supported by the National Natural Science Foundation of China (31901342), the Fundamental Research Funds for the Central Nonprofit Research Institution of Chinese Academy of Forestry (CAFYBB2020ZY003), and the key R\&D and Promotion of He'nan Province of China (192102110063).

T.W. and L.W. are the corresponding authors. E-mail: wanglin1815@163.com or tanatanan@163.com.

This is an open access article distributed under the CC BY-NC-ND license (https://creativecommons.org/licenses/by-nc-nd/4.0/).
(Sharma et al., 2010) and biodiesel fuel (Wang et al., 2017). They are also used in traditional Chinese medicine.

NAC proteins are plant-specific transcription factors that contain an N-terminal DNA-binding domain [no apical meristem $(N A M)$ ], a nuclear localization signal sequence [Arabidopsis transcription activation factor $(A T A F 1 / 2)$ ], and a C-terminal transcriptional activation domain [cup-shaped cotyledon (CUC2)] (Hu et al., 2015b; Ooka et al., 2003; Shen et al., 2020). The Nterminal DNA-binding domain usually contains $\approx 150$ amino acid residues and is further divided into five subdomains (A to E) (Apweiler et al., 2001; Duval et al., 2002; Kikuchi et al., 2000). The diverse C-terminal domain contains a transcriptional regulatory region (TRR) that activates or represses transcriptional activity (Delessert et al., 2005; Fang et al., 2008; Kim et al., 2007; Yamaguchi et al., 2010).

The NAC gene family has been intensively studied in various plant species since its discovery. In monocotyledons, 138 and 115 NAC genes have been identified in rice [Oryza sativa (Fang et al., 2008)] and maize [Zea mays (Zhu et al., 2012)], respectively. In dicotyledons, there are $105 \mathrm{NAC}$ genes in arabidopsis 


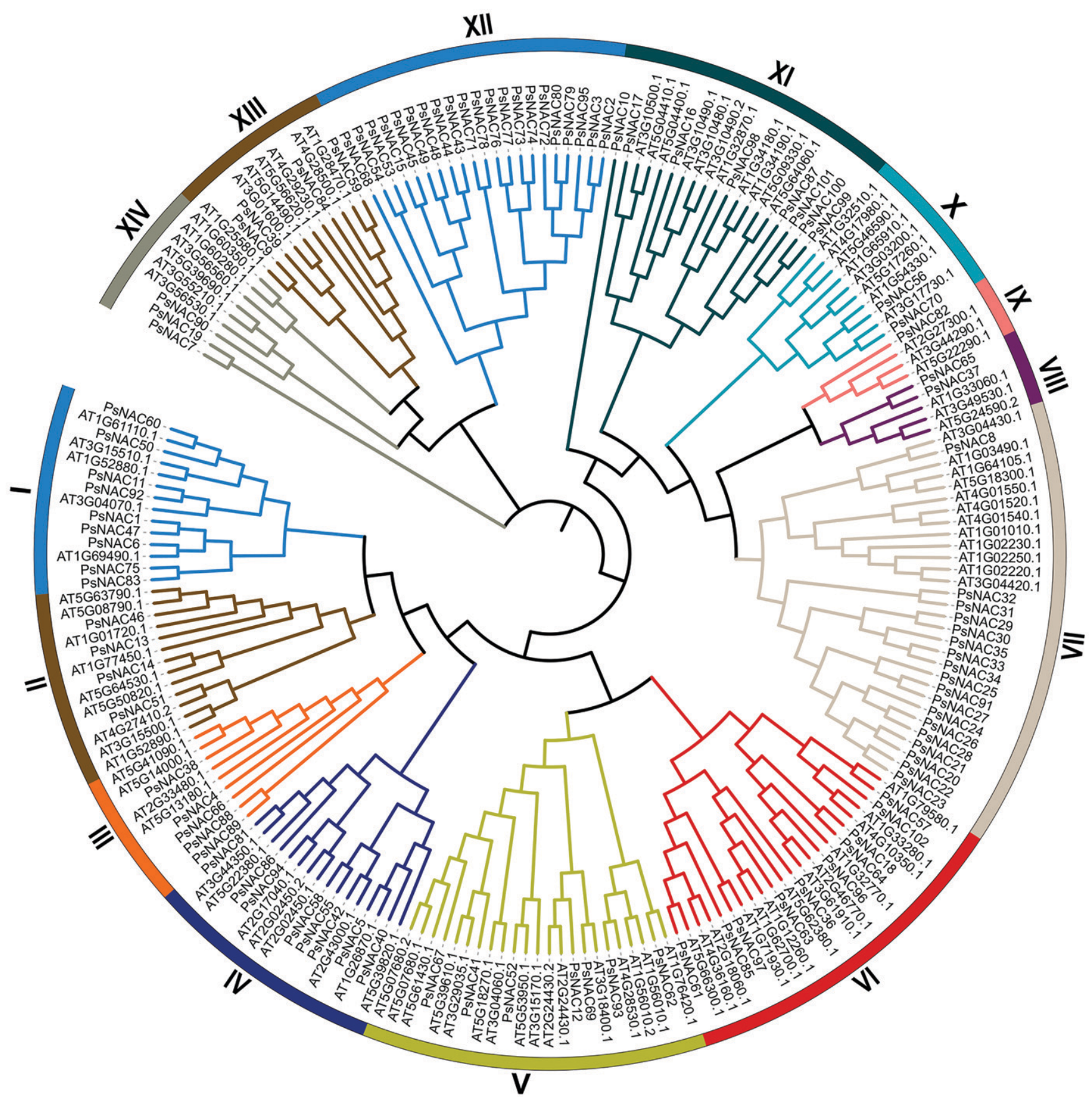

Fig. 1. Phylogenetic tree of the NAC genes in siberian apricot. Phylogenetic analysis of NAC genes from siberian apricot and arabidopsis using the complete protein sequences. Each of the 14 clades is indicated by a specific color.

[Arabidopsis thaliana (Ooka et al., 2003)], 74 in grape [Vitis vinifera (Wang et al., 2013)], 96 in cassava [Manihot esculenta (Hu et al., 2015b)], 93 in tomato [Solanum lycopersicum (Jin et al., 2020)], and 88 in pigeonpea [Cajanus cajan (Satheesh et al., 2014)]. In the Rosaceae, there are 180 NAC genes in apple [Malus domestica (Su et al., 2013)], 183 in white pear [Pyrus bretschneideri (Gong et al., 2019)], 114 in peach [Prunus persica (Gong et al., 2019)], and 113 in japanese apricot [Prunus mume (Zhuo et al., 2018)]. However, studies of the NAC gene family in siberian apricot are still limited (García-Gómez et al., 2019; Niu et al., 2016).

Previous reports have described the multiple functions of NAC transcription factors in plants, for example, controlling leaf senescence in arabidopsis (Guo and Gan, 2006), responding to ABA hormone signaling in arabidopsis (Jensen et al., 2008), forming secondary walls in woody plants (Mitsuda et al., 2007), controlling the growth transition between stamens and petals in arabidopsis (Sablowski and Meyerowitz, 1998), and regulating 
A

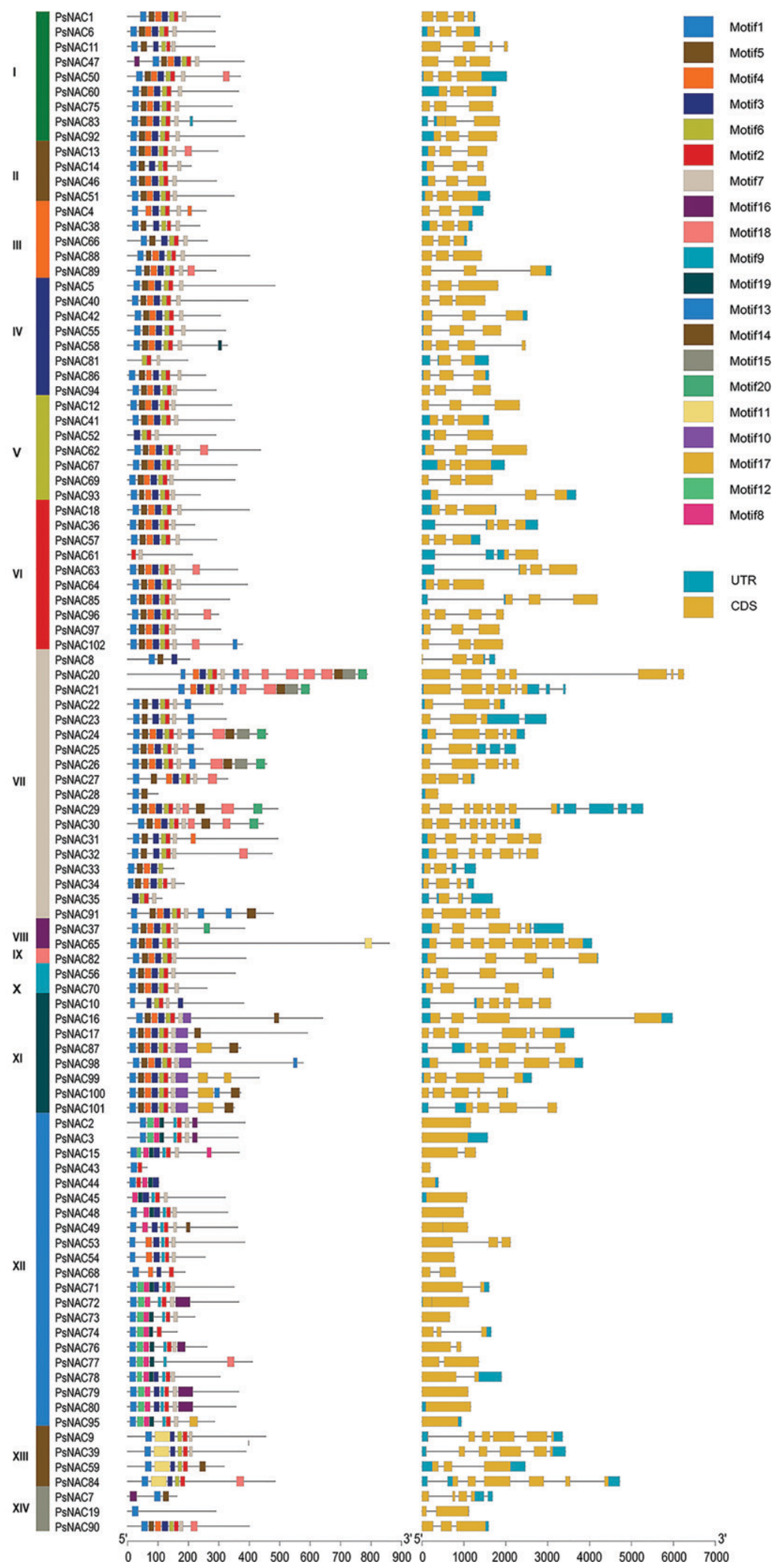

Fig. 2. The conserved motifs and gene structures of PsNACs. (A) The motif composition of 102 PsNACs. (B) Exon/intron structures of PSNACs. The motifs, numbered 1 to 20, are displayed in boxes with different colors. The sequence information for each motif is provided in Supplemental Fig. 1. The length of the protein could be estimated using the scale at the bottom. Green boxes indicate untranslated $5^{\prime}$ and $3^{\prime}$ regions; yellow boxes indicate exons; black lines indicate introns. shoot branching in rice (Mao et al., 2007). Additionally, the NAC gene family has crucial roles in fruit and seed development. For example, 13 NAC genes in cucumber ( $\mathrm{Cucu}$ mis sativus) impact fruit spine development (Liu et al., 2018). Solanum lycopersicum NAC gene 4 (SINAC4), a tomato NAC domain protein, accumulates to high levels at the onset of fruit ripening (Zhu et al., 2014). No-ripening-like 1 (NOR-like1), a NAC transcription factor, is a positive regulator of tomato fruit ripening (Gao et al., 2018). Six strawberry (Fragaria $\times$ ananassa) NAC proteins have been identified that have important regulatory roles in fruit development and ripening (Moyano et al., 2018). AtNAM is specifically expressed in developing arabidopsis seeds (Duval et al., 2002).

The availability of the siberian apricot genome is a valuable resource for the identification of gene families that allows us to gain further insight into their functions. Here, we report that the siberian apricot NAC gene (PSNAC) family may impact plant development, especially during fruit and kernel development. This study provides information regarding the functional characteristics and role of PSNACs in the development of siberian apricot.

\section{Materials and Methods}

IDENTIFICATION OF NAC FAMILY GENES. The hidden Markov model (HMM) file of the NAC domain (PF 02365) was downloaded from the Pfam protein family database (El-Gebali et al., 2019) to identify NAC family genes in siberian apricot genome (Genome Sequence Archive PRJCA001987) using HMMER 3.3 software (default para meters) (Finn et al., 2011) with a cutoff of 0.01. All candidate proteins were submitted to the National Center for Biotechnology Information Conserved Domain Database (Marchler-Bauer et al., 2017) and Simple Modular Architecture Research Tool (Letunic and Bork, 2018) to confirm the presence and completeness of the NAM domain. The molecular weights, isoelectric point, and subcellular localization tools were predicted on the ExPasy website (Gasteiger et al., 2003). The positions of all NAC family gene from the siberian apricot genome general feature format file were detected using TBtools program (Chen et al., 2018).

Phylogenetic tree analysis. NAC protein sequences from siberian apricot (102) and arabidopsis (105) were used to construct the phylogenetic tree. We performed multiple sequence alignment 


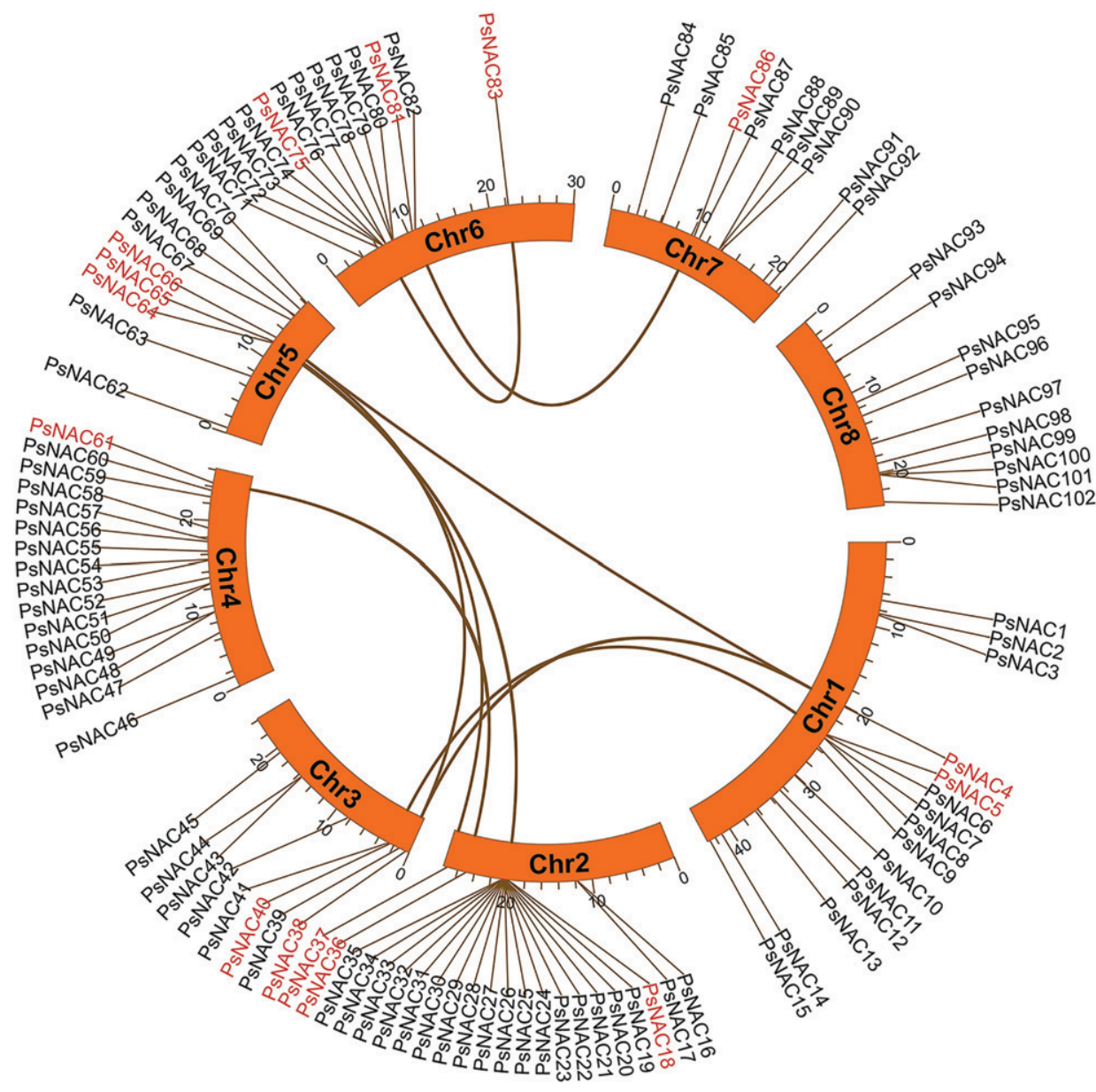

Fig. 3. Distribution and segmental duplication of $P S N A C$ s. Orange lines represent the chromosomes of siberian apricot. The scale on the chromosomes represents the chromosome length. The PsNACs in red are involved in duplication events. The brown lines indicate duplicated NAC gene pairs.

using MUSCLE (Edgar, 2004) set at the default parameters and constructed phylogenetic trees by using a maximum likelihood approach with 1000 bootstrap replicates using MEGA $\mathrm{X}$ (Kumar et al., 2018). The classification of PSNACs was performed according to their phylogenetic relationship with corresponding NAC genes of arabidopsis. The phylogenetic tree was displayed using the online tool iTOL (version 4) (Letunic and Bork, 2016).

Gene STRuCtures ANd CONSERved motifs of PsNACs. The gene structures of each PSNAC was drawn using the Gene Structure Display Server online software package (Hu et al., 2015a). The MEME tool (Bailey et al., 2009) was used to identify conserved motifs shared among siberian apricot NAC proteins (PsNACs) (Bailey and Gribskov, 1998; Bailey et al., 2009). The analysis parameters were set as follows: maximum number of different motifs, 20; minimum motif width, 6; and maximum motif width, 50 .

GeNe duPliCATION AND EVOLUTIONARY ANALYSIS OF PSNACs. We used the Multiple Collinearity Scan Toolkit (MCScanX, default parameters) (Wang et al., 2012) to examine whole-genome duplication (WGD) or segmental, tandem, and dispersed duplicates in the PSNACs. We analyzed the collinear relationship of the NAC genes of siberian apricot and peach, japanese apricot, and apple (Gong et al., 2019) using a dual synteny plotter (Chen et al.,
2018). Synonymous substitution (Ks) values were used to calculate the dates of duplication events $(\mathrm{T})$ according to the following equation: $\mathrm{T}=\mathrm{Ks} / 2 \lambda$, where $\lambda=1.5 \times 10^{-8}$ for dicots (Blanc and Wolfe, 2004; Koch et al., 2000). The mode of selection was identified according to the standard protocol described by Kayum using the nonsynonymous to synonymous substitutions $(\mathrm{Ka} / \mathrm{Ks})$ value, where $\mathrm{Ka} / \mathrm{Ks}<1$ indicates a negative selection, $\mathrm{Ka} / \mathrm{Ks}=1$ indicates a neutral selection, and $\mathrm{Ka} /$ Ks $>1$ indicates a positive selection (Gong et al., 2019).

EXPRESSION ANALYSIS USING RNASEQUENCING DATA. RNA-sequencing (RNA-seq) data were used to gain insight into the temporal and spatial transcription patterns and were obtained from the Genome Sequence Archive (PRJCA001987). Leaf, flower bud, and flower of the siberian apricot were collected on 15 Mar., 20 Mar., and 5 Apr. 2016, respectively. The fruit and kernel were collected on 16 May, 26 May, 5 June, 15 June, 25 June, and 5 July 2017, representing $25 \mathrm{~d}(\mathrm{~S} 1), 35 \mathrm{~d}$ (S2), 45 d (S3), 55 d (S4), 65 d (S5), and $75 \mathrm{~d}$ (S6) after full bloom, respectively. Two biological replicates for each sample were used. The expression levels of genes were measured as fragments per kilobase million (FPKM). The heat maps were created by Heatmap (Chen et al., 2018) based on the transformed data of $\log 2$ $(\mathrm{FPKM}+1)$ values.

Quantitative ReAl-Time POlyMerase Chain REACTION. For quantitative real-time polymerase chain reaction (qRTPCR) analysis, $1 \mu \mathrm{g}$ of DNA-free RNA was transcribed into first-strand complementary DNA (cDNA) using All-inOne First-Strand Synthesis MasterMix with dsDNase (CodonX Biotechnology Co., Beijing, China). The qRT-PCR was performed with a fluorescent quantitative PCR instrument (LightCycler 480; Roche Diagnostics, Mannheim, Germany) using $2 \times$ SYBR Green qPCR Premix Universal (CodonX Biotechnology Co.). The reaction conditions were 45 cycles at $95^{\circ} \mathrm{C}$ for $15 \mathrm{~s}, 60^{\circ} \mathrm{C}$ for $15 \mathrm{~s}$, and $60^{\circ} \mathrm{C}$ for $35 \mathrm{~s}$. The primer sequences of target genes were designed with Primer 3 software (Untergasser et al., 2012) and listed in Supplemental Table 1. The housekeeping siberian apricot Ubiquitin-conjugating enzyme (UBC) gene (Niu et al., 2014) was used as an internal control (Supplemental Table 1). Each reaction was performed with three repeats from different biological samples. The data from real-time PCR amplification were analyzed using the $2^{-\Delta \Delta C t}$ method. Statistical analysis using Student's $t$ test was performed with Microsoft Excel (version 2010 for Windows; Microsoft Corp., Redmond, WA). Data are provided as means \pm SD of three 
A

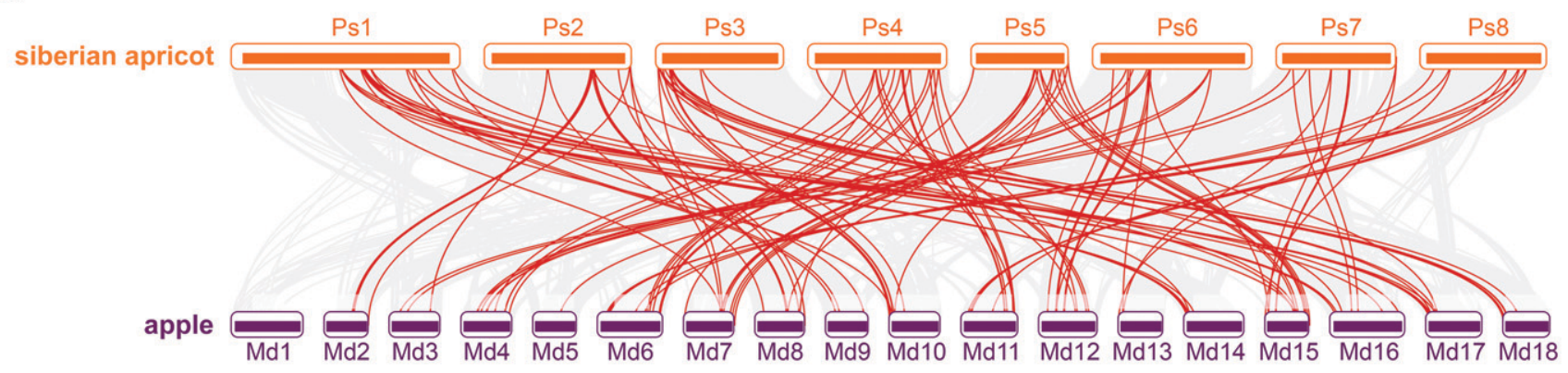

B

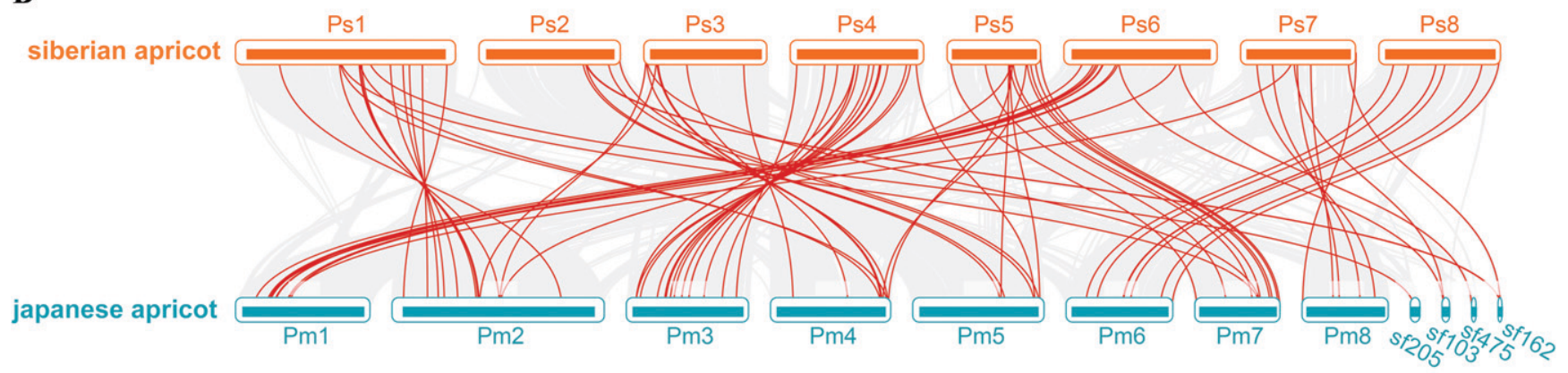

C

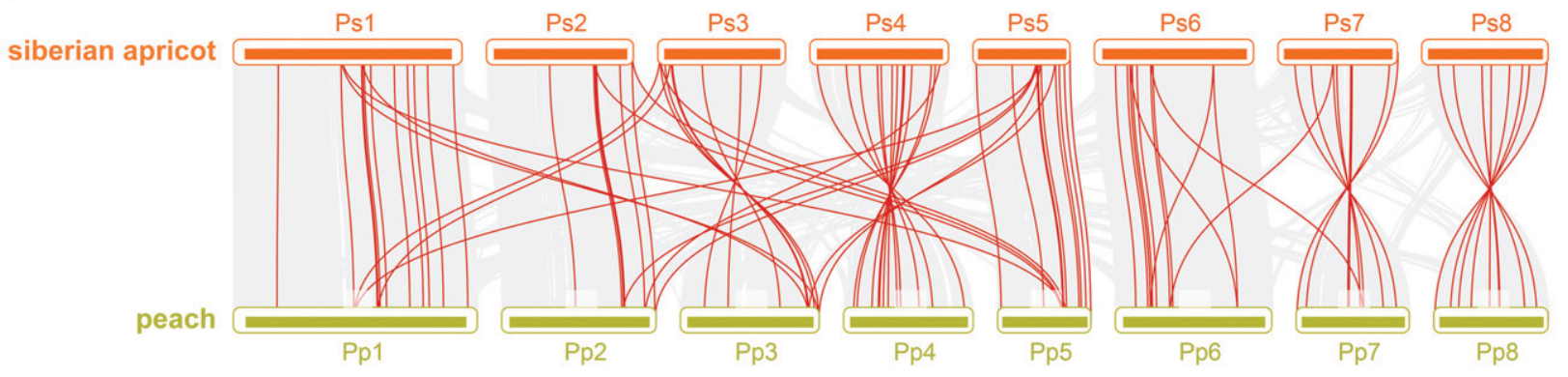

Fig. 4. Synteny analysis of NAC genes between siberian apricot and three Rosaceae species: (A) apple, (B) peach, and (C) japanese apricot. Orange, purple, blue, and green blocks present chromosomes of siberian apricot (Ps1-8), apple (Md1-18), japanese apricot (Pm1-8), and peach (Pp1-8), respectively. Red lines denote syntenic NAC gene pairs.

independent biological replicates. The Pearson correlation value, linear fitting equation, and $R^{2}$ values of the RNA-seq data and qRT-PCR were assumed using IBM SPSS Statistics (version 23; IBM Corp., Armonk, NY).

\section{Results}

IDENTIFICATION OF PSNACS IN SIBERIAN APRICOT GENOME. A total of 102 putative NAC protein genes were identified in siberian apricot genome and named after their locations on the chromosomes (PsNAC1-PsNAC102) (Supplemental Table 2). The lengths of the predicted proteins ranged from 64 to 860 amino acids (aa), with isoelectric point values between 4.38 and 9.80 and molecular weights between 7.52 and $95.98 \mathrm{kDa}$. Subcellular localization prediction indicated that most of the PsNACs (90) are located in the nucleus, whereas only single PsNACs were predicted to be localized in the plasma membrane (PsNAC28), vacuole (PsNAC17), and chloroplast (PsNAC67). Two PsNACs were predicted to localize in the mitochondrion (PsNACs 35 and 44), three were predicted to localize in the extracellular space (PsNACs 11, 19, and 43), and four were predicted to localize in the cytoplasm (PsNACs 34, 87, 93, and 99) (Supplemental Table 2).

Phylogenetic analysis and classification of PsNaCs. Based on the homology of the PsNACs with those from arabidopsis, 105 AtNACs and 102 PsNACs were grouped into 14 phylogenetic clades: I (NAP); II (AtNAC3/ATAF); III (SENU5); IV (ONAC22/TERN); V (NAC1/NAM); VI (OsNAC7); VII (ANAC001); VIII (TIP); IX (OsNAC8); X (ANAC011); XI (NAC2); XII and XIII (ONAC003); and XIV (ANAC063) (Fig. 1). Clade XII contained the largest number of PsNACs (21), followed by clades VII (18) and VI (11). Clade IX had the fewest members, with only one PsNAC. Additionally, the proteins in clade XII were found to be specific to siberian apricot (Supplemental Table 3). 


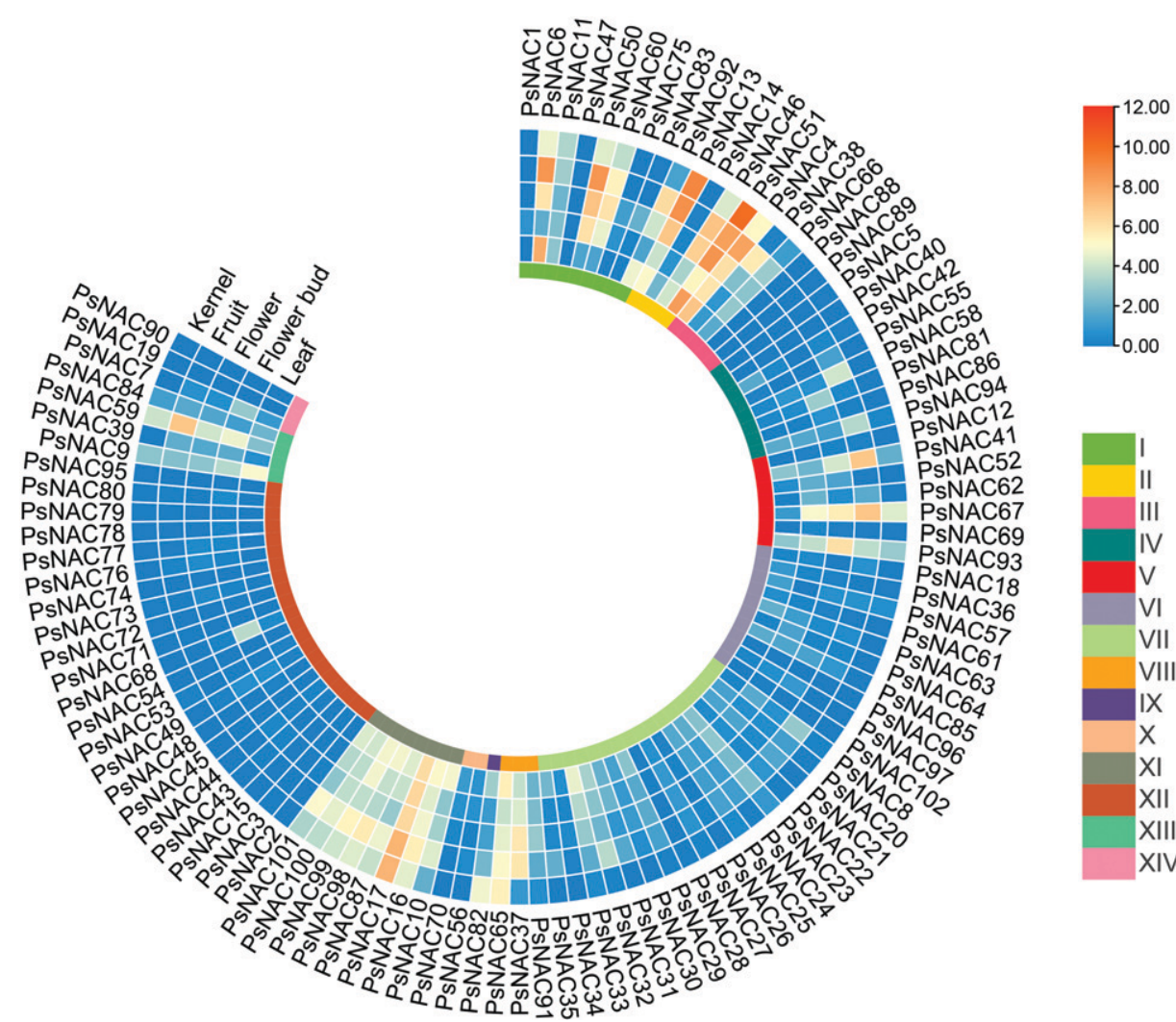

Fig. 5. Gene expressions of PsNACs in leaf, flower bud, flower, fruit, and kernel. RNA-sequencing (RNA-seq) expressions of fruit and kernel are from $75 \mathrm{~d}$ after full bloom (S6). Color scale of the upper right represents the value in fragments per kilobase million (FPKM).

Predicted protein motifs and structures of PsNACs. To understand the structural diversity of the PSNACs, we determined the conserved protein motifs in the predicted PsNACs and the exon/intron composition of the genes (Fig. 2). A total of 20 conserved motifs were identified (Fig. 2A, Supplemental Fig. 1), and all of the PsNACs contained at least one conserved motif (motifs 1-7). Specifically, motifs 1 to 7 occurred in a specific order (motifs 1, 5, 4, 3, 6, 2, and 7) in the proteins because they represent the conserved subdomains A, B, C1, C2, D1, D2, and $\mathrm{E}$, respectively, of the N-terminal NAC domains. Except for clade XII, most of the conserved motifs were found in the N-terminal regions of the PsNACs, indicating their importance to the function of the NAC proteins. In addition, the motifs present in clade VII and clade XII proteins (13) were the most diverse. We found that all of the members of clade XI contained motifs 4, 5, and 10, except for PsNAC10. Motifs 8 and 15 were only absent from proteins in clades XII and VII, respectively. Motifs 4 and 5 were absent from all of the proteins in clade XIII. To further study the gene structures, we predicted the exon/intron composition in the 102 PSNACs. We found that the numbers of introns ranged from 0 to 11 (Fig. 2B, Supplemental Table 2$)$. The majority $(40 ; 39.22 \%)$ of the genes contained 2 introns, followed by 3 introns $(20 ; 19.61 \%), 0$ introns (11; $10.78 \%), 1$ intron $(10 ; 9.80 \%), 4$ introns $(7 ; 6.86 \%), 5$ introns $(6 ; 5.88 \%), 6$ introns $(4 ; 3.92 \%), 7$ introns $(2 ; 1.96 \%), 8$ introns (1; $0.98 \%)$, and 11 introns $(1 ; 0.98 \%)$. There were no PsNACs with 9 or 10 introns. Most of the PSNACs within a clade had similar exon/intron compositions; for example, all members of clades I, II, III, IV, and V had two introns except for PSNAC1, PSNAC11, PsNAC58, and PsNAC83. In contrast, members of clade VII had highly variable intron numbers, ranging from 0 to 11 . The conserved motifs and gene structures analyses suggested that PsNACs in the same clades may have similar biological functions.

Chromosomal LOCATION AND SYNTENy ANalysis of PsNACs The 102 $P S N A C$ s were unevenly distributed on the eight siberian apricot chromosomes (Fig. 3). Chromosome 2 had the most PSNACs $(22 ; 21.57 \%)$, followed by chromosome $4(16 ; 15.69 \%)$ and chromosome 1 (15; 14.71\%), whereas, only eight PSNACs were predicted to be located on chromosome 3 (7.84\%) (Fig. 3, Supplemental Table 2).

To identify any potential duplication events in PSNACs, we performed a collinearity analysis. Nine pairs of segmentally duplicated genes were found in the 102 PsNACs. Segmentally duplicated genes were located on chromosomes 1 to 7. Seven duplicated gene pairs were from the same clade, except PSNAC4 (clade XI) and PsNAC38 (clade II) and PsNAC38 (clade II) and PsNAC66 (clade XI) (Fig. 3). The nonsynonymous substitution $(\mathrm{Ka})$ and $\mathrm{Ks}$ ratios were calculated to estimate the selection pressure and divergence times of the gene duplication events. All $\mathrm{Ka} / \mathrm{Ks}$ ratios were $<1$ (Supplemental Table 4), which indicated that purifying selection was the main force behind the evolution of PSNACs. The divergence times of PSNACs in siberian apricot ranged from 104.77 to 3.49 million years ago, and most of the duplication events traced back to 40 to 80 million years ago (Supplemental Table 4).

We further performed synteny analyses to compare the siberian apricot genome with apple, peach, and japanese apricot. The results identified 129 pairs of homologous genes between 69 PSNACs from siberian apricot and 109 NAC genes in apple (Fig. 4A), followed by 101 pairs between siberian apricot (79) and peach (80) (Fig. 4B) and 86 pairs between siberian apricot (70) and japanese apricot (70) (Fig. 4C) (Supplemental Tables 5-7).

The divergence times of NAC genes in apple ranged from 86.59 to 7.42 million years ago, and most of them occurred 10 to 30 million years ago based on duplication events; for peach, the divergence times ranged from 110.81 to 0.76 million years ago, and most of them occurred between 1 and 10 million years ago. For japanese apricot, the divergence times ranged from 108.65 to 0.13 million years ago, with most of them occurring from 0.3 to 6 million years ago (Supplemental Tables 5-7). Comparisons of the divergence times among the four species showed the shortest time of speciation occurred in japanese apricot and siberian apricot, thereby providing further evidence of the close evolutionary relationship between these two species.

EXPRESSION PATTERNS OF PSNACS IN FIVE PLANT TISSUES. The expression patterns of $P S N A C$ s were investigated in leaves, 


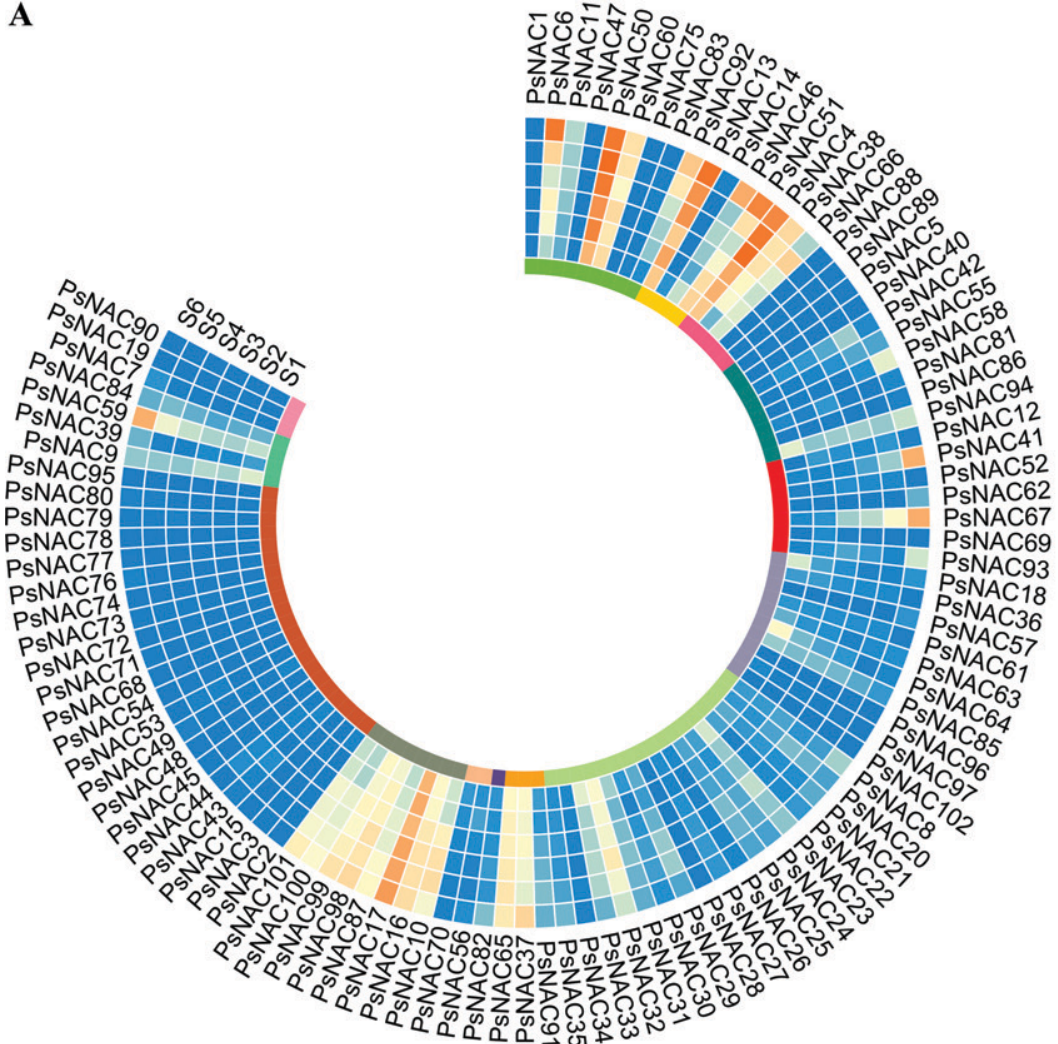

B

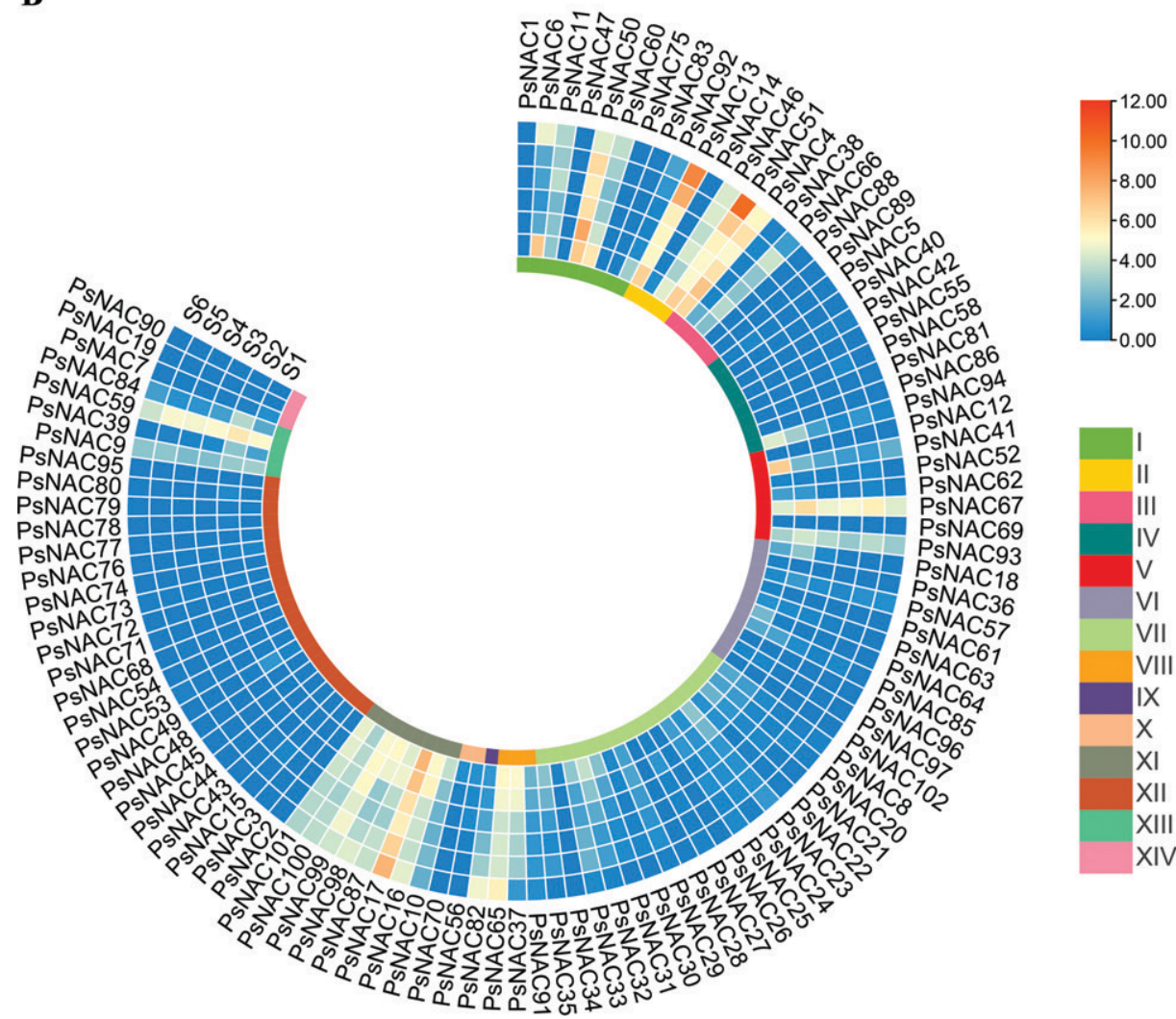

Fig. 6. Gene expression of PSNACs during (A) fruit and (B) kernel development. Color scale of the upper right represents the scale value in fragments per kilobase million (FPKM). flower buds, flowers, fruit, and kernels. Most PsNACs showed diverse expression patterns within each clade, indicating that these genes may have different functions in all tested tissues (Fig. 5). The expressions of clade VIII and clade XI genes were higher than those of genes from the other clades, indicating that genes in these clades may be involved in growth, development, and morphogenesis in the five tissues; each gene acquired a different function during evolution. PsNAC93 in clade $\mathrm{V}$ showed the highest expression level in the flower, whereas PsNAC41 and PSNAC67 were highly expressed in fruit. Additionally, the functions of the different clade proteins could be distinguished because most PSNACs had higher expression levels than others in the specific tissues: PSNAC6 (clade I), $P S N A C 33$ (clade VII), and PsNAC9 (clade XIII) in the leaf; PsNAC33 (clade VII) and PsNAC68 (clade XII) in the flower bud; and PsNAC93 (clade V) and PSNAC37 (clade VIII) in the flower. Eleven $P S N A C$ s were found to be highly expressed in the fruit, including PsNAC6, PsNAC50, and PsNAC92 (clade I), PsNAC13 (clade II), PsNAC4 and PSNAC38 (clade III), PSNAC41 and PsNAC67 (clade V), PsNAC37 (clade VIII), PsNAC17 (clade XI), and PsNAC59 (clade XIII). Four genes were highly expressed in the kernel, including PSNAC13 (clade II), PsNAC51 (clade II), PsNAC82 (clade IX), and PsNAC17 (clade XI). The expression patterns of all the aforementioned PsNACs, which were from clades I to III, V, VII, IX, and XI to XIII, showed overlap in the various tissues, indicating that PSNACs from these clades may have diverse functions.

EXPRESSION OF PSNACS DURING FRUIT AND KERNEL DEVELOPMENT. The expression levels of $P_{S} N A C$ s during fruit development were evaluated (Fig. 6A). Twenty-six PsNACs showed constitutive expression (FPKM $>1$ in all tested samples). Eight of the 26 genes from clade XI showed a general trend in which gene expression continued to increase during fruit development, indicating that these eight genes could have similar functions. Some PsNACs showed diverse functions based on the different expression patterns from the same clade. For example, the expression of PSNAC9 from clade XIII peaked at $\mathrm{S} 1$ during all stages of fruit 
development, whereas PsNAC59, also from clade XIII, showed the opposite expression pattern.

Eight PSNACs were highly expressed in S6, including PsNAC6 (clade I), PsNAC13, PsNAC46, and PsNAC51 (clade II), PsNAC41 and PsNAC67 (clade V), PsNAC37 (clade VIII), and $P S N A C 59$ (clade XIII), suggesting these $P S N A C$ s are associated with fruit maturation. Five $P S N A C \mathrm{~s}$, including $P S N A C 18$ and PsNAC64 (clade VI), PsNAC23 and PSNAC33 (clade VII), and $P S N A C 9$ (clade XIII), are highly expressed in $\mathrm{S} 1$; therefore, they may have important roles in fruit growth. The expression levels of PSNAC4 (clade III) peaked in S4 and PSNAC50 (clade I) in S5, respectively, suggesting that they may be involved in fruit development.

The expression levels of the PsNACs during kernel development were evaluated (Fig. 6B). The expression patterns in the kernel of PSNACs from most clades showed similar trends in the fruit; for example, genes from clade XI were the only group that showed constitutive expression, which suggests that clade XI genes have crucial roles in fruit and kernel development. Four $P S N A C$ s were found to be highly expressed in $\mathrm{S} 1$, including PsNAC6 (clade I), PsNAC94 (clade IV), PsNAC41 (clade V), and $P S N A C 32$ (clade VII), and these genes may regulate the rapid growth of the kernel. Three PsNACs expressed in S6, PSNAC82 (clade IX), and PSNAC13 and PSNAC51 (clade II) may impact dry matter accumulation and maturation of the kernel. PsNAC17 (clade XI) is highly expressed in both $\mathrm{S} 1$ and S6. Four PSNACs are highly expressed in S2, including PSNAC50 (clade I), PSNAC4 (clade III), PSNAC67 (clade V), and PsNAC84 (clade XIII), which indicated that these genes may affect the hardening of the kernel.

To further evaluate the validity of the RNA-seq data, 15 $P S A N C$ s were selected and their expressions were analyzed by qRT-PCR (Supplemental Fig. 2). The results showed that 14 of the 15 selected $P S N A C$ s had expression patterns that were consistent with those determined by RNA-seq (Supplemental Table 8). Therefore, the qRT-PCR results validated the reliability of the RNA-seq data.

\section{Discussion}

Characterization of the NAC gene family has been reported for many species in the Rosaceae, including strawberry (Moyano et al., 2018), apple (Su et al., 2013), white pear (Gong et al., 2019), peach (Gong et al., 2019), and japanese apricot (Zhuo et al., 2018), whereas studies of the NAC gene family in siberian apricot have provided unclear results (García-Gómez et al., 2019; Niu et al., 2016). In the present study, we systematically analyzed the NAC gene family in the siberian apricot genome. A total of 102 PSNACs were identified and divided into 14 clades based on the phylogenetic analysis; these results were similar to those of japanese apricot (Zhuo et al., 2018). This may be a result of the close evolutionary relationship between siberian apricot and japanese apricot. Interestingly, among the 14 PsNAC clades, the 21 members of clade XII had no corresponding AtNAC proteins, suggesting that the diversification and expansion of the PSNACs in clade XII occurred after the divergence of siberian apricot and arabidopsis.

Many of the PSNACs $(40 / 102 ; 39.22 \%)$ had 2 introns. These results are similar to those for japanese apricot $(48 / 113 ; 42.48 \%)$ (Zhuo et al., 2018), apple (79/180; 43.89\%) (Su et al., 2013), and white pear $(86 / 183 ; 46.99 \%)$ (Gong et al., 2019). Most of the PSNACs that encode proteins in the same clade showed similar exon/intron compositions, suggesting that the structures of PSNACs are relatively conserved.

Each PsNAC was predicted to contain at least one conserved motif (motifs 1-7). Motifs 1 to 7 correspond to subdomains A, $\mathrm{D} 2, \mathrm{C} 2, \mathrm{C} 1, \mathrm{~B}, \mathrm{D} 1$, and $\mathrm{E}$, respectively, which is consistent with previous findings for japanese apricot (Zhuo et al., 2018). Additionally, most of the conserved motifs were found in the $\mathrm{N}$-terminal regions of the predicted PsNACs, indicating that the $\mathrm{N}$-terminus is an important part of the NAC proteins, as has been shown previously for potato (Solanum tuberosum) (Koch et al., 2000) and cassava (Hu et al., 2015b). Therefore, our results provide evidence that the conserved motifs are significant to the functions of the NAC proteins.

Gene duplication events are an important way of generating new genes that encode proteins with improved or novel functions, and they are vital to the evolution of genomes and genetic systems (Moore and Purugganan, 2003). The recent occurrence of a WGD was found in apple and white pear (Su et al., 2013); in contrast, siberian apricot, peach, and japanese apricot showed evidence of an ancient WGD, but no recent WGD (Gong et al., 2019). Considering that most gene duplication events between japanese apricot and peach occurred $\approx 1$ to 5 million years ago (Zhuo et al., 2018), we inferred that siberian apricot has the closest evolutionary relationships with japanese apricot, followed by peach, and is evolutionarily most distant from apple.

We performed RNA-seq transcriptome analyses of five tissues to understand the expression patterns of the $P S N A C$ s and their physiological or reproductive functions. Three PSNACs had relatively higher expression levels in leaves, two in flower buds, two in flowers, four in kernels, and 11 (the highest number) during fruit development. These results suggest that the PSNACs may have acquired different functions during evolution. Similar tissue-specific expression profiles were found for other species, including tomato (Jin et al., 2020), cassava ( $\mathrm{Hu}$ et al., 2015b), and soybean (Le et al., 2011).

According to the RNA-seq analysis of the PSNACs involved in fruit and kernel development, we found that PsNAC4 (clade III) in S4, PsNAC50 (clade I) in S5, and $P S N A C 41$ (clade V) and PSNAC59 (clade XIII) in S6 were homologous with FvNAC042, FvNAC035, FvNAC092, and $F v N A C 006$ in strawberry, respectively; of these, $F v N A C 035$ impacts fruit ripening, $F v N A C 042$ is involved in fruit growth and development, and FvNAC006 and FvNAC092 may be related to fruit senescence (Moyano et al., 2018). These results imply that the functions of PsNACs may be related to the characteristics of the NAC gene family. PSNAC50 (clade I) and PsNAC67 (clade V) in S2 of kernel development are homologous with arabidopsis AtNAM (Duval et al., 2002) and maize $A P N-1$ (NAM-related protein 1-like) (Verza et al., 2011); AtNAM is associated with developing seeds and $A P N-1$ ( $N A M$-related protein 1-like) in maize has a role in regulating the late phases of endosperm development.

In summary, we identified and characterized the PSNAC gene/protein family in siberian apricot genome. We also performed systematic investigations of the gene structures and evolutionary relationships among the PSNACs and the proteins they are predicted to encode. Our results create a foundation for 
future research of the biological functions of PsNACs involved in fruit and kernel development in siberian apricot.

\section{Literature Cited}

Apweiler, R., T.K. Attwood, A. Bairoch, A. Bateman, E. Birney, M. Biswas, P. Bucher, L. Cerutti, F. Corpet, and M.D. Croning. 2001. The InterPro database, an integrated documentation resource for protein families, domains and functional sites. Nucleic Acids Res. 29(1):37-40, doi: 10.1093/nar/29.1.37.

Bailey, T.L., M. Boden, F.A. Buske, M. Frith, C.E. Grant, L. Clementi, J. Ren, W.W. Li, and W.S. Noble. 2009. MEME SUITE: Tools for motif discovery and searching. Nucleic Acids Res. 37(suppl_2):W202-W208, doi: 10.1093/nar/gkp335.

Bailey, T.L. and M. Gribskov. 1998. Combining evidence using p-values: Application to sequence homology searches. Bioinformatics 14(1):48-54, doi: 10.1093/bioinformatics/14.1.48.

Blanc, G. and K.H. Wolfe. 2004. Widespread paleopolyploidy in model plant species inferred from age distributions of duplicate genes. Plant Cell 16(7):1667-1678, doi: 10.1105/tpc.021345.

Chen, C., H. Chen, Y. He, R. Xia. 2018. TBtools, a toolkit for biologists integrating various biological data handling tools with a userfriendly interface. BioRxiv. 289660, doi: 10.1101/289660.

Delessert, C., K. Kazan, I.W. Wilson, D.V.D. Straeten, J. Manners, E.S. Dennis, and R. Dolferus. 2005. The transcription factor ATAF2 represses the expression of pathogenesis-related genes in arabidopsis. Plant J. 43(5):745-757, doi: 10.1111/j.1365-313X.2005.02488.x.

Duval, M., T.-F. Hsieh, S.Y. Kim, and T.L. Thomas. 2002. Molecular characterization of AtNAM: A member of the Arabidopsis NAC domain superfamily. Plant Mol. Biol. 50(2):237-248, doi: 10.1023/ A: 1016028530943.

Edgar, R.C. 2004. MUSCLE: Multiple sequence alignment with high accuracy and high throughput. Nucleic Acids Res. 32(5):1792-1797, doi: 10.1093/nar/gkh340.

El-Gebali, S., J. Mistry, A. Bateman, S.R. Eddy, A. Luciani, S.C. Potter, M. Qureshi, L.J. Richardson, G.A. Salazar, and A. Smart. 2019. The Pfam protein families database in 2019. Nucleic Acids Res. 47(D1):D427-D432, doi: 10.1093/nar/gky995.

Fang, Y., J. You, K. Xie, W. Xie, and L. Xiong. 2008. Systematic sequence analysis and identification of tissue-specific or stress-responsive genes of NAC transcription factor family in rice. Mol. Genet. Genomics 280(6):547-563, doi: 10.1007/s00438-008-0386-6.

Finn, R.D., J. Clements, and S.R. Eddy. 2011. HMMER web server: Interactive sequence similarity searching. Nucleic Acids Res. 39(suppl_2):W29-W37, doi: 10.1093/nar/gkr367.

Gao, Y., W. Wei, X. Zhao, X. Tan, Z. Fan, Y. Zhang, Y. Jing, L. Meng, B. Zhu, and H. Zhu. 2018. A NAC transcription factor, NORlike 1 , is a new positive regulator of tomato fruit ripening. Hort. Res. 5(1):1-18, doi: 10.1038/s41438-018-0111-5.

García-Gómez, B.E., J.A. Salazar, L. Dondini, P. Martínez-Gómez, and D. Ruiz. 2019. Identification of QTLs linked to fruit quality traits in apricot (Prunus armeniaca L.) and biological validation through gene expression analysis using qPCR. Mol. Breed. 39(2):28, doi: 10.1007/s11032-018-0926-7.

Gasteiger, E., A. Gattiker, C. Hoogland, I. Ivanyi, R.D. Appel, and A. Bairoch. 2003. ExPASy: The proteomics server for in-depth protein knowledge and analysis. Nucleic Acids Res. 31(13):3784-3788, doi: 10.1093/nar/gkg563.

Gong, X., L. Zhao, X. Song, Z. Lin, B. Gu, J. Yan, S. Zhang, S. Tao, and $X$. Huang. 2019. Genome-wide analyses and expression patterns under abiotic stress of NAC transcription factors in white pear (Pyrus bretschneideri). BMC Plant Biol. 19(1):1-18, doi: 10.1186/s12870019-1760-8.

Guo, Y. and S. Gan. 2006. AtNAP, a NAC family transcription factor, has an important role in leaf senescence. Plant J. 46(4):601-612, doi: 10.1111/j.1365-313X.2006.02723.x.
Hu, B., J. Jin, A.-Y. Guo, H. Zhang, J. Luo, and G. Gao. 2015a. GSDS 2.0: An upgraded gene feature visualization server. Bioinformatics 31(8):1296-1297, doi: 10.1093/bioinformatics/btu817.

Hu, W., Y. Wei, Z. Xia, Y. Yan, X. Hou, M. Zou, C. Lu, W. Wang, and M. Peng. 2015b. Genome-wide identification and expression analysis of the NAC transcription factor family in cassava. PLoS One 10(8):e0136993, doi: 10.1186/s12864-019-5500-0.

Jensen, M.K., P.H. Hagedorn, M. De Torres-Zabala, M.R. Grant, J.H. Rung, D.B. Collinge, and M.F. Lyngkjaer. 2008. Transcriptional regulation by an NAC (NAM-ATAF1, 2-CUC2) transcription factor attenuates ABA signalling for efficient basal defence towards Blumeria graminis $f$. sp. hordei in arabidopsis. Plant J. 56(6):867-880, doi: 10.1111/j.1365-313X.2008.03646.x.

Jin, J.F., Z.Q. Wang, Q.Y. He, J.Y. Wang, P.F. Li, J.M. Xu, S.J. Zheng, W. Fan, and J.L. Yang. 2020. Genome-wide identification and expression analysis of the NAC transcription factor family in tomato (Solanum lycopersicum) during aluminum stress. BMC Genomics 21:1-14, doi: 10.1186/s12864-020-6689-7.

Kikuchi, K., M. Ueguchi-Tanaka, K. Yoshida, Y. Nagato, M. Matsusoka, and H.-Y. Hirano. 2000. Molecular analysis of the NAC gene family in rice. Mol. Gen. Genet. 262(6):1047-1051, doi: $10.1007 /$ PL00008647.

Kim, H.S., B.O. Park, J.H. Yoo, M.S. Jung, S.M. Lee, H.J. Han, K.E. Kim, S.H. Kim, C.O. Lim, and D.-J. Yun. 2007. Identification of a calmodulin-binding NAC protein as a transcriptional repressor in arabidopsis. J. Biol. Chem. 282(50):36292-36302, doi: 10.1074/ jbc.M705217200.

Koch, M.A., B. Haubold, and T. Mitchell-Olds. 2000. Comparative evolutionary analysis of chalcone synthase and alcohol dehydrogenase loci in Arabidopsis, Arabis, and related genera (Brassicaceae). Mol. Biol. Evol. 17(10):1483-1498, doi: 10.1093/oxfordjournals.molbev.a026248.

Kumar, S., G. Stecher, M. Li, C. Knyaz, and K. Tamura. 2018. MEGA X: Molecular evolutionary genetics analysis across computing platforms. Mol. Biol. Evol. 35(6):1547-1549, doi: 10.1093/molbev/msy096.

Le, D.T., R. Nishiyama, Y. Watanabe, K. Mochida, K. YamaguchiShinozaki, K. Shinozaki, and L.-S.P. Tran. 2011. Genome-wide survey and expression analysis of the plant-specific NAC transcription factor family in soybean during development and dehydration stress. DNA Res. 18(4):263-276, doi: 10.1093/dnares/dsr015.

Letunic, I. and P. Bork. 2016. Interactive tree of life (iTOL) v3: An online tool for the display and annotation of phylogenetic and other trees. Nucleic Acids Res. 44(W1):W242-W245, doi: 10.1093/nar/ gkw290.

Letunic, I. and P. Bork. 2018. 20 years of the SMART protein domain annotation resource. Nucleic Acids Res. 46(D1):D493-D496, doi: 10.1093/nar/gkx922.

Liu, X., E. Bartholomew, K. Black, M. Dong, Y. Zhang, S. Yang, Y. Cai, S. Xue, Y. Weng, and H. Ren. 2018. Comprehensive analysis of NAC transcription factors and their expression during fruit spine development in cucumber (Cucumis sativus L.). Hort. Res. 5(1):1-14, doi: 10.1038/s41438-018-0036-z.

Mao, C., W. Ding, Y. Wu, J. Yu, X. He, H. Shou, and P. Wu. 2007. Overexpression of a NAC-domain protein promotes shoot branching in rice. New Phytol. 176(2):288-298, doi: 10.1111/j.14698137.2007.02177.x.

Marchler-Bauer, A., Y. Bo, L. Han, J. He, C.J. Lanczycki, S. Lu, F. Chitsaz, M.K. Derbyshire, R.C. Geer, and N.R. Gonzales. 2017. CDD/SPARCLE: Functional classification of proteins via subfamily domain architectures. Nucleic Acids Res. 45(D1):D200-D203, doi: 10.1093/nar/gkw1129.

Mitsuda, N., A. Iwase, H. Yamamoto, M. Yoshida, M. Seki, K. Shinozaki, and M. Ohme-Takagi. 2007. NAC transcription factors, NST1 and NST3, are key regulators of the formation of secondary walls in woody tissues of arabidopsis. Plant Cell 19(1):270-280, doi: $10.1105 /$ tpc. 106.047043 . 
Moore, R.C. and M.D. Purugganan. 2003. The early stages of duplicate gene evolution. Proc. Natl. Acad. Sci. USA 100(26):15682-15687, doi: 10.1073/pnas.2535513100.

Moyano, E., F.J. Martínez-Rivas, R. Blanco-Portales, F.J. Molina-Hidalgo, P. Ric-Varas, A.J. Matas-Arroyo, J.L. Caballero, J. Muñoz-Blanco, and A. Rodríguez-Franco. 2018. Genome-wide analysis of the NAC transcription factor family and their expression during the development and ripening of the Fragaria $\times$ ananassa fruits. PLoS One 13(5):e0196953, doi: 10.1371/journal.pone.0196953.

Niu, J., J. Wang, J. An, L. Liu, Z. Lin, R. Wang, L. Wang, C. Ma, L. Shi, and S. Lin. 2016. Integrated mRNA and miRNA transcriptome reveal a cross-talk between developing response and hormone signaling for the seed kernels of Siberian apricot. Sci. Rep. 6(1):1-12, doi: $10.1038 /$ srep35675.

Niu, J., B. Zhu, J. Cai, P. Li, L. Wang, H. Dai, L. Qiu, H. Yu, D. Ha, and H. Zhao. 2014. Selection of reference genes for gene expression studies in siberian apricot (Prunus sibirica L.) germplasm using quantitative real-time PCR. PLoS One 9(8):e103900, doi: 10.1371/ journal.pone. 0103900 .

Ooka, H., K. Satoh, K. Doi, T. Nagata, Y. Otomo, K. Murakami, K. Matsubara, N. Osato, J. Kawai, and P. Carninci. 2003. Comprehensive analysis of NAC family genes in Oryza sativa and Arabidopsis thaliana. DNA Res. 10(6):239-247, doi: 10.1093/dnares/10.6.239.

Sablowski, R.W. and E.M. Meyerowitz. 1998. A homolog of NO APICAL MERISTEM is an immediate target of the floral homeotic genes APETALA3/PISTILLATA. Cell 92(1):93-103, doi: 10.1016/ S0092-8674(00)80902-2.

Satheesh, V., P.T.K. Jagannadham, P. Chidambaranathan, P. Jain, and R. Srinivasan. 2014. NAC transcription factor genes: Genome-wide identification, phylogenetic, motif and cis-regulatory element analysis in pigeonpea (Cajanus cajan (L.) Millsp.). Mol. Biol. Rep. 41(12):7763-7773, doi: 10.1007/s11033-014-3669-5.

Sharma, P., B. Tilakratne, and A. Gupta. 2010. Utilization of wild apricot kernel press cake for extraction of protein isolate. J. Food Sci. Technol. 47(6):682-685, doi: 10.1007/s13197-010-0096-Z.

Shen, S., Q. Zhang, Y. Shi, Z. Sun, Q. Zhang, S. Hou, R. Wu, L. Jiang, X. Zhao, and Y. Guo. 2020. Genome-wide analysis of the NAC domain transcription factor gene family in Theobroma cacao. Genes (Basel) 11(1):35, doi: 10.3390/genes11010035.

Su, H., S. Zhang, X. Yuan, C. Chen, X.-F. Wang, and Y.-J. Hao. 2013. Genome-wide analysis and identification of stress-responsive genes of the NAM-ATAF1, 2-CUC2 transcription factor family in apple. Plant Physiol. Biochem. 71:11-21, doi: 10.1016/j.plaphy.2013.06.022.

Untergasser, A., I. Cutcutache, T. Koressaar, J. Ye, B.C. Faircloth, M. Remm, and S.G. Rozen. 2012. Primer3-new capabilities and interfaces. Nucleic Acids Res. 40(15):e115, doi: 10.1093/nar/ gks596.

Verza, N., T. Figueira, S. Sousa, and P. Arruda. 2011. Transcription factor profiling identifies an aleurone-preferred NAC family member involved in maize seed development. Ann. Appl. Biol. 158(1):115-129, doi: 10.1111/j.1744-7348.2010.00447.x.

Wang, L. and H. Yu. 2012. Biodiesel from siberian apricot (Prunus sibirica L.) seed kernel oil. Bioresour. Technol. 112:355-358, doi: 10.1016/j.biortech.2012.02.120.

Wang, N., Y. Zheng, H. Xin, L. Fang, and S. Li. 2013. Comprehensive analysis of NAC domain transcription factor gene family in Vitis vinifera. Plant Cell Rep. 32(1):61-75, doi: 10.1007/s00299-0121340-y.

Wang, Y., H. Tang, J.D. DeBarry, X. Tan, J. Li, X. Wang, T.-h. Lee, H. Jin, B. Marler, and H. Guo. 2012. MCScanX: A toolkit for detection and evolutionary analysis of gene synteny and collinearity. Nucleic Acids Res. 40(7):e49, doi: 10.1093/nar/gkr1293.

Wang, Z., Y. Zeng, Z. Zhang, S. Sheng, J. Tian, R. Wu, and X. Pang. 2017. Phylogeography study of the siberian apricot (Prunus sibirica L.) in Northern China assessed by chloroplast microsatellite and DNA makers. Front. Plant Sci. 8:1989, doi: 10.3389/fpls.2017.01989.

Yamaguchi, M., M. Ohtani, N. Mitsuda, M. Kubo, M. Ohme-Takagi, H. Fukuda, and T. Demura. 2010. VND-INTERACTING2, a NAC domain transcription factor, negatively regulates xylem vessel formation in arabidopsis. Plant Cell 22(4):1249-1263, doi: 10.1105/ tpc.108.064048.

Zhang, J., Z. Wei, and H. Zhao. 2006. Determination of ability of several arbor and shrub species to endure and survive extreme aridity with limited-areas methods under field conditions in Horqin Sandy Land. Acta Ecol. Sin. 26(2):467-474, doi: 10.1016/S18722032(06)60010-3.

Zhu, M., G. Chen, S. Zhou, Y. Tu, Y. Wang, T. Dong, and Z. Hu. 2014. A new tomato NAC (N AM/A TAF1/2/C UC2) transcription factor, SINAC4, functions as a positive regulator of fruit ripening and carotenoid accumulation. Plant Cell Physiol. 55(1):119-135, doi: $10.1093 / \mathrm{pcp} / \mathrm{pct} 162$.

Zhu, T., E. Nevo, D. Sun, and J. Peng. 2012. Phylogenetic analyses unravel the evolutionary history of NAC proteins in plants. Evolution: Intl. J. of Organic Evolution. 66(6):1833-1848, doi: 10.1111/ j.1558-5646.2011.01553.x.

Zhuo, X., T. Zheng, Z. Zhang, Y. Zhang, L. Jiang, S. Ahmad, L. Sun, J. Wang, T. Cheng, and Q. Zhang. 2018. Genome-wide analysis of the NAC transcription factor gene family reveals differential expression patterns and cold-stress responses in the woody plant Prunus mume. Genes (Basel) 9(10):494, doi: 10.3390/genes9100494. 
Supplemental Table 1. Primer sequences used for quantitative real-time polymerase chain reaction (qRT-PCR).

\begin{tabular}{|c|c|c|}
\hline Gene name & Sense primer & Anti-sense primer \\
\hline$\overline{U B C^{\mathrm{Z}}}$ & GAGACCAGCAATAACCGTGAA & TCTTGTACTCCGTGGCATCCT \\
\hline PSNAC9 & GATGTTCGCTGGCACAAGACT & ССТTСТСАТССТССТСАGTTCC \\
\hline PsNAC59 & TGGCACAAGACAGGGAAGACAA & ACTTTTGAGACCACCAGCTCCC \\
\hline PsNAC33 & CTTGGGATTTACCTGGGATGTT & CTCGCTTAGTGACCCTGTTGG \\
\hline PsNAC51 & CTCTGGTTTTCTATGTCGGCAA & ACGACACAAAACCCATTCATCC \\
\hline PsNAC67 & GTCTGAGGACAAACAGGGCAAC & GCTCTGCCTCTGTAGAAAACCAAG \\
\hline PsNAC37 & GGTCTTCCATACTGGTCGTGCT & CAGACGACAAAGAACAAAGGGAT \\
\hline PsNAC13 & ACAAATACAACCCCTGGGACCT & CCAAAGCCTTCTTAATCCCGACT \\
\hline PsNAC50 & CAGGGACTGATAAGCCCGTGT & TGGTCTTGTTATCAGCCAGCCTA \\
\hline PsNAC64 & AGAATCGGGTTGAGAAAGACGC & ATACTCGGCATACCACCCACC \\
\hline PsNAC94 & CGTTTGCCTGATAACTGCCACT & GTTGGCTGCAAAATGAGATGGT \\
\hline PsNAC32 & TTTAGGGATACTTCCGCTGACG & CGGCAGTTTGAGGATATGGAGG \\
\hline
\end{tabular}

${ }^{\mathrm{z}} U B C=$ Ubiquitin-conjugating enzyme gene.

Supplemental Table 2. Information for NAC genes in siberian apricot genome.

\begin{tabular}{|c|c|c|c|c|c|c|c|c|c|c|c|}
\hline Gene name & Gene & Chromosome & Start & End & Strand & $\mathrm{aa}^{\mathrm{z}}$ & $\mathrm{CDS}^{\mathrm{z}}$ & Exon no. & $\mathrm{pI}^{\mathrm{z}}$ & $\mathrm{MW}^{\mathrm{z}}$ & Cell location \\
\hline$\overline{P s N A C l}$ & PaF106G0100001051.01.T02 & Chr1 & 6804341 & 6805605 & - & 304 & 911 & 4 & 5.94 & 34608.19 & Nucleus \\
\hline PsNAC2 & PaF106G0100001251.01.T01 & Chr1 & 8404432 & 8405592 & - & 386 & 1160 & 1 & 4.76 & 44225.08 & Nucleus \\
\hline$P S N A C 3$ & PaF106G0100001279.01.T01 & Chr1 & 8567150 & 8568717 & + & 363 & 1091 & 1 & 5.01 & 41486.18 & Nucleus \\
\hline PsNAC4 & PaF106G0100002691.01.T01 & Chr1 & 20140448 & 20141909 & - & 258 & 774 & 3 & 9.16 & 29439.4 & Nucleus \\
\hline PsNAC5 & PaF106G0100003290.01.T01 & Chr1 & 23997571 & 23999385 & + & 484 & 1452 & 3 & 7.26 & 53697.01 & Nucleus \\
\hline PsNAC6 & PaF106G0100003324.01.T01 & Chr1 & 24162587 & 24163966 & - & 288 & 864 & 3 & 7.01 & 33193.55 & Nucleus \\
\hline PsNAC7 & PaF106G0100003398.01.T02 & Chr1 & 24487653 & 24489336 & - & 162 & 485 & 5 & 5.04 & 18457.53 & Nucleus \\
\hline PsNAC8 & PaF106G0100003399.01.T02 & Chr1 & 24494068 & 24495808 & - & 204 & 612 & 4 & 5.17 & 23330.42 & Nucleus \\
\hline PSNAC9 & PaF106G0100003636.01.T01 & Chr1 & 25687665 & 25691020 & + & 454 & 1359 & 6 & 4.84 & 51532.22 & Nucleus \\
\hline PSNAC10 & PaF106G0100004524.01.T01 & Chr1 & 30089751 & 30092820 & + & 382 & 1144 & 6 & 7.91 & 42409.48 & Nucleus \\
\hline PsNAC11 & PaF106G0100005052.01.T03 & Chr1 & 32558219 & 32560259 & + & 287 & 860 & 4 & 9.36 & 33034.65 & Extracellular \\
\hline PsNAC12 & PaF106G0100005288.01.T01 & Chr1 & 33802255 & 33804582 & + & 342 & 1026 & 3 & 8.95 & 38086.85 & Nucleus \\
\hline PsNAC13 & PaF106G0100005713.01.T02 & Chr1 & 36334023 & 36335570 & + & 297 & 891 & 3 & 8.09 & 34149.33 & Nucleus \\
\hline PsNACl4 & PaF106G0100006471.01.T01 & Chr1 & 41134752 & 41136214 & + & 209 & 627 & 3 & 5.15 & 23852.47 & Nucleus \\
\hline PsNAC15 & PaF106G0100006718.01.T01 & Chr1 & 42716774 & 42718051 & + & 367 & 1102 & 2 & 4.85 & 40933.42 & Nucleus \\
\hline PsNAC16 & PaF106G0200008014.01.T01 & Chr2 & 11802179 & 11808156 & + & 641 & 1922 & 4 & 5.79 & 69822.65 & Nucleus \\
\hline PSNAC17 & PaF106G0200008016.01.T04 & Chr2 & 11821460 & 11825086 & + & 591 & 1770 & 6 & 4.51 & 66277.37 & Vacuole \\
\hline PsNAC18 & PaF106G0200009314.01.T01 & $\mathrm{Chr} 2$ & 20032319 & 20034084 & - & 400 & 1200 & 4 & 5.92 & 45208.13 & Nucleus \\
\hline PSNAC19 & PaF106G0200009318.01.T01 & Chr2 & 20062396 & 20063511 & - & 290 & 871 & 2 & 4.8 & 33108.47 & Extracellular \\
\hline PsNAC20 & PaF106G0200009362.01.T03 & Chr2 & 20317704 & 20323951 & - & 788 & 2360 & 7 & 5.05 & 84824.2 & Nucleus \\
\hline PsNAC21 & PaF106G0200009364.01.T02 & $\mathrm{Chr} 2$ & 20326120 & 20329544 & - & 598 & 1791 & 8 & 5.06 & 64473.71 & Nucleus \\
\hline PsNAC22 & PaF106G0200009366.01.T01 & Chr2 & 20332537 & 20334505 & - & 313 & 939 & 3 & 5.37 & 34380.65 & Nucleus \\
\hline PsNAC23 & PaF106G0200009367.01.T01 & $\mathrm{Chr} 2$ & 20335987 & 20338951 & - & 324 & 972 & 4 & 5.51 & 35736.17 & Nucleus \\
\hline PsNAC24 & PaF106G0200009369.01.T01 & $\mathrm{Chr} 2$ & 20342925 & 20345373 & - & 460 & 1378 & 5 & 4.69 & 51859.21 & Nucleus \\
\hline PsNAC25 & PaF106G0200009371.01.T01 & $\mathrm{Chr} 2$ & 20348339 & 20350573 & - & 248 & 744 & 5 & 8.32 & 28128.51 & Nucleus \\
\hline PsNAC26 & PaF106G0200009372.01.T01 & $\mathrm{Chr} 2$ & 20355733 & 20358036 & - & 457 & 1369 & 5 & 4.51 & 51503.89 & Nucleus \\
\hline PsNAC27 & PaF106G0200009374.01.T02 & Chr2 & 20362068 & 20363313 & - & 329 & 987 & 3 & 8.68 & 38064.08 & Nucleus \\
\hline PsNAC28 & PaF106G0200009376.01.T01 & Chr2 & 20401724 & 20402105 & - & 100 & 302 & 1 & 5.21 & 11283.31 & Plasma membrane \\
\hline PSNAC29 & PaF106G0200009380.01.T01 & Chr2 & 20437996 & 20443271 & - & 494 & 1477 & 12 & 5.04 & 57082.2 & Nucleus \\
\hline PsNAC30 & PaF106G0200009381.01.T02 & Chr2 & 20445394 & 20447732 & - & 446 & 1332 & 9 & 4.62 & 51962.39 & Nucleus \\
\hline PSNAC31 & PaF106G0200009383.01.T01 & $\mathrm{Chr} 2$ & 20449886 & 20452725 & - & 494 & 1479 & 6 & 6.79 & 57559.45 & Nucleus \\
\hline PsNAC32 & PaF106G0200009384.01.T06 & $\mathrm{Chr} 2$ & 20459703 & 20462470 & - & 475 & 1421 & 7 & 7.21 & 55305.04 & Nucleus \\
\hline PsNAC33 & PaF106G0200009404.01.T01 & Chr2 & 20567897 & 20569176 & + & 152 & 456 & 4 & 9.76 & 17941.81 & Nucleus \\
\hline PsNAC34 & PaF106G0200009406.01.T01 & Chr2 & 20571304 & 20572535 & + & 186 & 557 & 4 & 5.22 & 21887.67 & Cytoplasm \\
\hline PsNAC35 & PaF106G0200009409.01.T01 & $\mathrm{Chr} 2$ & 20589188 & 20590871 & + & 113 & 340 & 4 & 9.8 & 13442.35 & Mitochondrion \\
\hline PsNAC36 & PaF106G0200010296.01.T01 & $\mathrm{Chr} 2$ & 24966612 & 24969376 & - & 221 & 663 & 4 & 8.56 & 26134.61 & Nucleus \\
\hline PsNAC37 & PaF106G0200010786.01.T02 & $\mathrm{Chr} 2$ & 27262191 & 27265562 & - & 385 & 1153 & 6 & 5.17 & 43566.53 & Nucleus \\
\hline PsNAC38 & PaF106G0300010962.01.T01 & Chr3 & 430522 & 431723 & - & 237 & 711 & 3 & 9.38 & 27123.66 & Nucleus \\
\hline PsNAC39 & PaF106G0300011153.01.T01 & Chr3 & 1275832 & 1279258 & - & 389 & 1164 & 6 & 5.18 & 42813.48 & Nucleus \\
\hline PsNAC40 & PaF106G0300011420.01.T01 & $\mathrm{Chr} 3$ & 2467978 & 2469483 & - & 395 & 1185 & 3 & 6.02 & 44068.17 & Nucleus \\
\hline PsNAC41 & PaF106G0300011468.01.T01 & Chr3 & 2652398 & 2653995 & + & 352 & 1056 & 3 & 6.56 & 40144.96 & Nucleus \\
\hline PsNAC42 & PaF106G0300012473.01.T01 & Chr3 & 8261982 & 8264494 & + & 305 & 915 & 3 & 8.35 & 35325.99 & Nucleus \\
\hline PsNAC43 & PaF106G0300013351.01.T01 & Chr3 & 15562474 & 15562668 & + & 64 & 194 & 1 & 4.44 & 7517.43 & Extracellular \\
\hline PsNAC44 & PaF106G0300013354.01.T01 & Chr3 & 15588895 & 15589284 & + & 103 & 311 & 1 & 6.57 & 11759.22 & Mitochondrion \\
\hline PsNAC45 & PaF106G0300013852.01.T01 & Chr3 & 19401906 & 19402978 & - & 321 & 965 & 1 & 4.92 & 35761.65 & Nucleus \\
\hline PsNAC46 & PaF106G0400014754.01.T01 & Chr4 & 1314360 & 1315884 & - & 292 & 876 & 3 & 6.19 & 33694.09 & Nucleus \\
\hline PsNAC47 & PaF106G0400015194.01.T01 & Chr4 & 6757960 & 6759582 & + & 383 & 1149 & 3 & 8.28 & 44502.24 & Nucleus \\
\hline PsNAC48 & PaF106G0400015523.01.T01 & Chr4 & 9268251 & 9269240 & - & 329 & 989 & 1 & 4.88 & 36895.89 & Nucleus \\
\hline PsNAC49 & PaF106G0400015525.01.T01 & $\mathrm{Chr} 4$ & 9280734 & 9281824 & - & 362 & 1087 & 2 & 5.02 & 41186.2 & Nucleus \\
\hline PsNAC50 & PaF106G0400015969.01.T01 & Chr4 & 12618287 & 12620308 & - & 371 & 1113 & 3 & 7.18 & 41158.12 & Nucleus \\
\hline
\end{tabular}


Supplemental Table 2. (Continued)

\begin{tabular}{|c|c|c|c|c|c|c|c|c|c|c|c|}
\hline Gene name & Gene & Chromosome & Start & End & Strand & $\mathrm{aa}^{\mathrm{z}}$ & $\mathrm{CDS}^{\mathrm{z}}$ & Exon no. & $\mathrm{pI}^{\mathrm{z}}$ & $\mathrm{MW}^{\mathrm{z}}$ & Cell location \\
\hline$\overline{P s N A C 51}$ & PaF106G0400015972.01.T01 & Chr4 & 12640569 & 12642193 & - & 350 & 1050 & 3 & 8.14 & 39213.97 & Nucleus \\
\hline PsNAC52 & PaF106G0400016087.01.T01 & Chr4 & 13324581 & 13326270 & - & 290 & 871 & 3 & 8.8 & 31870.59 & Nucleus \\
\hline PsNAC53 & PaF106G0400016422.01.T01 & Chr4 & 15293662 & 15295771 & + & 385 & 1155 & 3 & 8.41 & 43665.14 & Nucleus \\
\hline PsNAC54 & PaF106G0400016439.01.T01 & Chr4 & 15405087 & 15405854 & - & 255 & 767 & 1 & 5.47 & 29722.26 & Nucleus \\
\hline PSNAC55 & PaF106G0400016619.01.T01 & Chr4 & 16375152 & 16377035 & - & 322 & 966 & 3 & 6.67 & 37184.41 & Nucleus \\
\hline PsNAC56 & PaF106G0400016793.01.T01 & Chr4 & 17500303 & 17503440 & - & 354 & 1061 & 4 & 4.77 & 40320.58 & Nucleus \\
\hline PsNAC57 & PaF106G0400016834.01.T02 & Chr4 & 17751495 & 17752879 & - & 293 & 879 & 3 & 7.04 & 33458.48 & Nucleus \\
\hline PsNAC58 & PaF106G0400017046.01.T01 & Chr4 & 19111398 & 19113864 & + & 328 & 983 & 4 & 8.97 & 37526.37 & Nucleus \\
\hline PsNAC59 & PaF106G0400017722.01.T01 & $\mathrm{Chr} 4$ & 22771175 & 22773638 & - & 317 & 951 & 3 & 8.47 & 35771.22 & Nucleus \\
\hline PsNAC60 & PaF106G0400017892.01.T01 & $\mathrm{Chr} 4$ & 23532747 & 23534514 & + & 365 & 1095 & 3 & 8.76 & 40148.64 & Nucleus \\
\hline PsNAC61 & PaF106G0400018075.01.T01 & Chr4 & 24441507 & 24444272 & + & 213 & 640 & 4 & 5.7 & 24282.87 & Nucleus \\
\hline PsNAC62 & PaF106G0500018516.01.T01 & Chr5 & 631098 & 633597 & + & 437 & 1311 & 3 & 6.33 & 49275.31 & Nucleus \\
\hline PsNAC63 & PaF106G0500019383.01.T01 & Chr5 & 7301548 & 7305242 & - & 362 & 1086 & 4 & 6.4 & 42228.94 & Nucleus \\
\hline PsNAC64 & PaF106G0500020061.01.T01 & Chr5 & 11974628 & 11976100 & - & 394 & 1182 & 3 & 5.94 & 45450.45 & Nucleus \\
\hline PsNAC65 & PaF106G0500020096.01.T01 & Chr5 & 12160028 & 12164081 & - & 860 & 2575 & 8 & 5.04 & 95982.39 & Nucleus \\
\hline PsNAC66 & PaF106G0500020210.01.T01 & Chr5 & 12737137 & 12738204 & - & 262 & 786 & 3 & 8.21 & 29696.28 & Nucleus \\
\hline PsNAC67 & PaF106G0500020796.01.T01 & Chr5 & 15443847 & 15445816 & + & 360 & 1080 & 3 & 7.64 & 40281.49 & Chloroplast \\
\hline PsNAC68 & PaF106G0500020907.01.T02 & Chr5 & 16125215 & 16126014 & + & 189 & 568 & 2 & 7.79 & 21790.59 & Nucleus \\
\hline PsNAC69 & PaF106G0500021114.01.T01 & Chr5 & 17089806 & 17091486 & + & 353 & 1059 & 3 & 6.61 & 39611.23 & Nucleus \\
\hline PsNAC70 & PaF106G0500021325.01.T01 & Chr5 & 18137845 & 18140144 & - & 261 & 783 & 3 & 5.07 & 29853.16 & Nucleus \\
\hline PsNAC7I & PaF106G0600022049.01.T02 & Chr6 & 3943738 & 3945341 & + & 350 & 1051 & 2 & 5.15 & 39864.78 & Nucleus \\
\hline PsNAC72 & PaF106G0600022464.01.T01 & Chr6 & 6149190 & 6150307 & + & 366 & 1099 & 2 & 4.71 & 42209.09 & Nucleus \\
\hline PsNAC73 & PaF106G0600022544.01.T01 & Chr6 & 6675482 & 6676147 & - & 221 & 665 & 1 & 8.54 & 26077.31 & Nucleus \\
\hline PsNAC74 & PaF106G0600022545.01.T01 & Chr6 & 6680851 & 6682501 & - & 163 & 489 & 3 & 8.79 & 19337.88 & Nucleus \\
\hline PsNAC75 & PaF106G0600022547.01.T01 & Chr6 & 6688780 & 6690470 & - & 344 & 1032 & 3 & 8.81 & 39185.23 & Nucleus \\
\hline PsNAC76 & PaF106G0600022604.01.T01 & Chr6 & 7085247 & 7086174 & + & 261 & 784 & 2 & 8.32 & 30169.42 & Nucleus \\
\hline PsNAC77 & PaF106G0600022607.01.T01 & Chr6 & 7094122 & 7095475 & + & 410 & 1231 & 2 & 4.38 & 46410.02 & Nucleus \\
\hline PsNAC78 & PaF106G0600022711.01.T01 & Chr6 & 7930679 & 7932577 & - & 304 & 913 & 2 & 5.68 & 34799.28 & Nucleus \\
\hline PsNAC79 & PaF106G0600022722.01.T01 & Chr6 & 7973329 & 7974426 & + & 365 & 1097 & 1 & 4.77 & 42331.88 & Nucleus \\
\hline PsNAC80 & PaF106G0600022733.01.T01 & Chr6 & 8064375 & 8065537 & - & 356 & 1070 & 2 & 4.69 & 41315.79 & Nucleus \\
\hline PsNAC81 & PaF106G0600023030.01.T01 & Chr6 & 10543449 & 10545038 & + & 198 & 595 & 3 & 6.19 & 21988.6 & Nucleus \\
\hline PsNAC82 & PaF106G0600023066.01.T01 & Chr6 & 10951041 & 10955242 & + & 389 & 1166 & 4 & 5.84 & 43480.38 & Nucleus \\
\hline PsNAC83 & PaF106G0600024369.01.T01 & Chr6 & 22291525 & 22293375 & + & 356 & 1068 & 4 & 7.55 & 40413.21 & Nucleus \\
\hline PsNAC84 & PaF106G0700026542.01.T01 & $\mathrm{Chr} 7$ & 3116989 & 3121707 & - & 485 & 1452 & 7 & 6.63 & 54591.53 & Nucleus \\
\hline PsNAC85 & PaF106G0700027143.01.T01 & Chr7 & 6153741 & 6157924 & - & 335 & 1005 & 4 & 5 & 38649.15 & Nucleus \\
\hline PsNAC86 & PaF106G0700027914.01.T01 & $\mathrm{Chr} 7$ & 10349543 & 10351137 & + & 257 & 771 & 3 & 9.64 & 29567.39 & Nucleus \\
\hline PsNAC87 & PaF106G0700028020.01.T02 & Chr7 & 10981600 & 10985010 & - & 372 & 1113 & 7 & 4.94 & 41460.6 & Cytoplasm \\
\hline PsNAC88 & PaF106G0700028379.01.T01 & $\mathrm{Chr} 7$ & 13627838 & 13629260 & + & 401 & 1203 & 3 & 6.32 & 46086.95 & Nucleus \\
\hline PsNAC89 & PaF106G0700028380.01.T01 & $\mathrm{Chr} 7$ & 13631687 & 13634769 & + & 290 & 870 & 3 & 5.9 & 33560.09 & Nucleus \\
\hline PsNAC90 & PaF106G0700028413.01.T01 & Chr7 & 13869695 & 13871283 & - & 400 & 1200 & 3 & 4.86 & 45270.23 & Nucleus \\
\hline PsNAC91 & PaF106G0700029093.01.T03 & Chr7 & 20891518 & 20893369 & - & 479 & 1436 & 4 & 5.09 & 53507.76 & Nucleus \\
\hline PsNAC92 & PaF106G0700029265.01.T01 & Chr7 & 22450132 & 22451918 & - & 384 & 1152 & 3 & 7.76 & 42977.5 & Nucleus \\
\hline PsNAC93 & PaF106G0800029902.01.T01 & Chr8 & 2990380 & 2994055 & - & 239 & 717 & 3 & 8.6 & 27565.43 & Cytoplasm \\
\hline PsNAC94 & PaF106G0800030484.01.T01 & Chr8 & 5916364 & 5917998 & - & 291 & 873 & 3 & 7.03 & 33868.74 & Nucleus \\
\hline PsNAC95 & PaF106G0800031447.01.T01 & Chr8 & 11262037 & 11262976 & + & 286 & 860 & 1 & 5.59 & 33051.85 & Nucleus \\
\hline PsNAC96 & PaF106G0800031681.01.T01 & Chr8 & 12998406 & 13000349 & + & 299 & 896 & 4 & 8.77 & 34038.83 & Nucleus \\
\hline PsNAC97 & PaF106G0800032053.01.T01 & Chr8 & 16472387 & 16474230 & - & 306 & 918 & 3 & 6.21 & 35519.6 & Nucleus \\
\hline PsNAC98 & PaF106G0800032290.01.T01 & Chr8 & 18879434 & 18883273 & + & 577 & 1729 & 5 & 5.1 & 65381.9 & Nucleus \\
\hline PsNAC99 & PaF106G0800032439.01.T01 & Chr8 & 20206705 & 20209321 & - & 432 & 1295 & 4 & 4.55 & 48114.89 & Cytoplasm \\
\hline PsNAC100 & PaF106G0800032441.01.T02 & Chr8 & 20212858 & 20214900 & - & 372 & 1114 & 5 & 5.01 & 41963.01 & Nucleus \\
\hline PsNAC101 & PaF106G0800032442.01.T01 & Chr8 & 20223298 & 20226512 & - & 351 & 1052 & 5 & 5.32 & 39292.02 & Nucleus \\
\hline PsNAClO2 & PaF106G0800032900.01.T01 & Chr8 & 23458676 & 23460601 & - & 378 & 1134 & 3 & 7.29 & 42968.71 & Nucleus \\
\hline
\end{tabular}

$\mathrm{z}_{\mathrm{aa}}=$ amino acids, $\mathrm{CDS}=$ coding sequence, $\mathrm{pI}=$ isoelectric point, $\mathrm{MW}=$ molecular weight. 
Supplemental Table 3. Grouping of NAC proteins in siberian apricot and arabidopsis.

\begin{tabular}{|c|c|c|}
\hline Clade & PsNAC & AtNAC \\
\hline I (NAP) & $\begin{array}{l}\text { PsNAC1, PsNAC6, PsNAC11, PsNAC47, } \\
\text { PsNAC50, PsNAC60, PsNAC75, } \\
\text { PsNAC83, PsNAC } 92\end{array}$ & $\begin{array}{l}\text { AT1G52880.1, AT3G15510.1, } \\
\text { AT1G61110.1, AT1G69490.1, } \\
\text { AT3G04070.1 }\end{array}$ \\
\hline II (AtNAC3/ATAF) & PsNAC51, PsNAC13, PsNAC46, PsNAC14 & $\begin{array}{l}\text { AT1G52890.1, AT3G15500.1, } \\
\text { AT4G27410.2, AT1G01720.1, } \\
\text { AT1G77450.1, AT5G08790.1, } \\
\text { AT5G63790.1, AT5G50820.1, } \\
\text { AT5G64530.1 }\end{array}$ \\
\hline III (SENU5) & $\begin{array}{l}\text { PsNAC66, PsNAC88, PsNAC89, PsNAC4, } \\
\text { PsNAC38 }\end{array}$ & $\begin{array}{l}\text { AT5G13180.1, AT2G33480.1, } \\
\quad \text { AT5G14000.1, AT5G41090.1 }\end{array}$ \\
\hline IV (ONAC22/TERN) & $\begin{array}{l}\text { PsNAC5, PsNAC40, PsNAC42, PsNAC55, } \\
\text { PsNAC58, PsNAC94, PsNAC81, } \\
\text { PsNAC } 86\end{array}$ & $\begin{array}{l}\text { AT5G39820.1, AT1G26870.1, } \\
\text { AT2G43000.1, AT2G17040.1, } \\
\text { AT2G02450.1, AT2G02450.2, } \\
\text { AT3G44350.1, AT5G22380.1 }\end{array}$ \\
\hline $\mathrm{V}(\mathrm{NAC} 1 / \mathrm{NAM})$ & $\begin{array}{l}\text { PsNAC12, PsNAC41, PsNAC52, } \\
\text { PsNAC62, PsNAC67, PsNAC69, } \\
\text { PsNAC } 93\end{array}$ & $\begin{array}{l}\text { AT5G07680.1, AT5G07680.2, } \\
\text { AT5G61430.1, AT3G29035.1, } \\
\text { AT5G39610.1, AT3G04060.1, } \\
\text { AT5G18270.1, AT1G76420.1, } \\
\text { AT5G53950.1, AT3G15170.1, } \\
\text { AT3G18400.1, AT2G24430.1, } \\
\text { AT2G24430.2, AT1G56010.1, } \\
\text { AT1G56010.2, AT4G28530.1 }\end{array}$ \\
\hline VI (OsNAC7) & $\begin{array}{l}\text { PsNAC18, PsNAC36, PsNAC57, } \\
\text { PsNAC61, PsNAC63, PsNAC64, } \\
\text { PsNAC } 85, \text { PsNAC96, PsNAC97, } \\
\text { PsNAC102 }\end{array}$ & $\begin{array}{l}\text { AT1G12260.1, AT1G62700.1, } \\
\text { AT5G62380.1, AT1G71930.1, } \\
\text { AT5G66300.1, AT2G18060.1, } \\
\text { AT4G36160.1, AT1G33280.1, } \\
\text { AT4G10350.1, AT1G79580.1, } \\
\text { AT1G32770.1, AT2G46770.1, } \\
\text { AT3G61910.1 }\end{array}$ \\
\hline VII (ANAC001) & $\begin{array}{l}\text { PsNAC20, PsNAC21, PsNAC22, } \\
\text { PsNAC23, PsNAC24, PsNAC25, } \\
\text { PsNAC26, PsNAC27, PsNAC28, } \\
\text { PsNAC29, PsNAC30, PsNAC31, } \\
\text { PsNAC32, PsNAC33, PsNAC34, } \\
\text { PsNAC 35, PsNAC91, PsNAC8 }\end{array}$ & $\begin{array}{l}\text { AT1G01010.1, AT1G02220.1, } \\
\text { AT3G04420.1, AT1G02230.1, } \\
\text { AT1G02250.1, AT4G01550.1, } \\
\text { AT4G01520.1, AT4G01540.1, } \\
\text { AT1G64105.1, AT5G18300.1, } \\
\text { AT3G04430.1, AT1G03490.1 }\end{array}$ \\
\hline VIII (TIP) & PsNAC37, PsNAC65 & $\begin{array}{l}\text { AT1G33060.1, AT3G49530.1, } \\
\text { AT5G24590.2 }\end{array}$ \\
\hline IX (OsNAC8) & PsNAC 82 & $\begin{array}{l}\text { AT3G44290.1, AT5G22290.1, } \\
\text { AT2G27300.1 }\end{array}$ \\
\hline X (ANAC011) & PsNAC56, PsNAC70 & $\begin{array}{l}\text { AT1G54330.1, AT3G17730.1, } \\
\text { AT1G65910.1, AT3G03200.1, } \\
\text { AT5G17260.1, AT1G32510.1, } \\
\text { AT4G17980.1, AT5G46590.1 }\end{array}$ \\
\hline $\mathrm{XI}(\mathrm{NAC} 2)$ & $\begin{array}{l}\text { PsNAC10, PsNAC16, PsNAC17, } \\
\text { PsNAC87, PsNAC98, PsNAC99, } \\
\text { PsNAC100, PsNAC101 }\end{array}$ & $\begin{array}{l}\text { AT5G09330.1, AT5G64060.1, } \\
\text { AT3G10500.1, AT5G04410.1, } \\
\text { AT5G04400.1, AT3G10480.1, } \\
\text { AT3G10490.1, AT3G10490.2, } \\
\text { AT1G34180.1, AT1G34190.1, } \\
\text { AT1G32870.1 }\end{array}$ \\
\hline XII & $\begin{array}{l}\text { PsNAC2, PsNAC3, PsNAC15, PsNAC43, } \\
\text { PsNAC44, PsNAC45, PsNAC48, } \\
\text { PsNAC49, PsNAC53, PsNAC54, } \\
\text { PsNAC68, PsNAC71, PsNAC72, } \\
\text { PsNAC73, PsNAC74, PsNAC76, } \\
\text { PsNAC77, PsNAC78, PsNAC79, } \\
\text { PsNAC80, PsNAC95 }\end{array}$ & \\
\hline
\end{tabular}


Supplemental Table 3. (Continued)

\begin{tabular}{lcc}
\hline Clade & PsNAC & AtNAC \\
\hline X III (ONAC003) & PsNAC9, PsNAC39, PsNAC59, PsNAC84 & AT1G28470.1, AT4G28500.1, \\
& & AT5G56620.1, AT4G29230.1, \\
& & AT1G25580.1, AT3G01600.1, \\
X IV (ANAC063) & AT5G14490.1 \\
& & AT3G56560.1, AT3G56530.1, \\
& & AT3G55210.1, AT5G39690.1, \\
\hline
\end{tabular}

Supplemental Table 4. Estimated non-synonymous (Ka) to synonymous (Ks) substitution ratios and divergence times of the duplicated PsNACs.

\begin{tabular}{|c|c|c|c|c|c|}
\hline Duplication gene pairs & $\mathrm{Ka}$ & Ks & $\mathrm{Ka} / \mathrm{Ks}$ & Selection type & $\begin{array}{c}\text { Divergence time } \\
(\mathrm{Mya})^{\mathrm{z}}\end{array}$ \\
\hline$\overline{P s N A C 4 \text { vs. } P s N A C 38}$ & 0.460 & 1.874 & 0.245 & Purifying & 62.48 \\
\hline PsNAC4 vs. PsNAC66 & 0.271 & 1.159 & 0.234 & Purifying & 38.63 \\
\hline PsNAC18 vs. PsNAC64 & 0.323 & 2.041 & 0.158 & Purifying & 68.02 \\
\hline PsNAC36 vs. PsNAC61 & 0.079 & 0.105 & 0.756 & Purifying & 3.49 \\
\hline PsNAC37 vs. PsNAC65 & 0.424 & 3.143 & 0.135 & Purifying & 104.77 \\
\hline PsNAC75 vs. PsNAC83 & 0.442 & 1.750 & 0.253 & Purifying & 58.34 \\
\hline$P s N A C 81$ vs. $P s N A C 86$ & 0.624 & 2.303 & 0.271 & Purifying & 76.78 \\
\hline
\end{tabular}

${ }^{\mathrm{z}}$ Million years ago. 
Supplemental Table 5. Estimated non-synonymous (Ka) to synonymous (Ks) substitution ratios and divergence times of the systenic gene pairs of NAC between siberian apricot and apple.

\begin{tabular}{|c|c|c|c|c|}
\hline Duplication gene pairs & $\mathrm{Ka}$ & Ks & $\mathrm{Ka} / \mathrm{Ks}$ & $\begin{array}{c}\text { Divergence time } \\
(\mathrm{Mya})^{\mathrm{z}}\end{array}$ \\
\hline PsNAC4 vs. $M D 06 G 1135700$ & 0.277 & 1.371 & 0.202 & 45.69 \\
\hline PsNAC4 vs. $M D 13 G 1125000$ & 0.117 & 0.667 & 0.175 & 22.23 \\
\hline PsNAC4 vs. $M D 14 G 1150900$ & 0.301 & 1.660 & 0.181 & 55.34 \\
\hline PsNAC4 vs. $M D 16 G 1125800$ & 0.073 & 0.542 & 0.134 & 18.07 \\
\hline PSNAC5 vs. $M D 09 G 1050400$ & 0.319 & 1.426 & 0.224 & 47.53 \\
\hline PsNAC5 vs. $M D 13 G 1072500$ & 0.076 & 0.386 & 0.197 & 12.87 \\
\hline PsNAC5 vs. $M D 16 G 1073300$ & 0.066 & 0.401 & 0.165 & 13.38 \\
\hline PsNAC6 vs. MD13G1069200 & 0.107 & 0.780 & 0.138 & 26.02 \\
\hline PsNAC6 vs. MD16G1069500 & 0.109 & 0.777 & 0.140 & 25.89 \\
\hline PsNAC7 vs. $M D 13 G 1063900$ & 0.395 & 0.868 & 0.454 & 28.94 \\
\hline PsNAC7 vs. $M D 16 G 1062600$ & 0.393 & 0.821 & 0.479 & 27.35 \\
\hline PsNAC9 vs. $M D 13 G 1046600$ & 0.088 & 0.242 & 0.365 & 8.06 \\
\hline PsNAC9 vs. $M D 16 G 1048300$ & 0.088 & 0.223 & 0.395 & 7.42 \\
\hline PsNAC11 vs. $M D 08 G 1096700$ & 0.207 & 0.507 & 0.408 & 16.89 \\
\hline PsNAC11 vs. $M D 15 G 1079400$ & 0.191 & 0.450 & 0.425 & 15.01 \\
\hline PsNAC12 vs. $M D 08 G 1121300$ & 0.075 & 0.350 & 0.215 & 11.66 \\
\hline PsNAC12 vs. $M D 15 G 1100100$ & 0.057 & 0.407 & 0.139 & 13.58 \\
\hline PsNAC13 vs. $M D 15 G 1344900$ & 0.078 & 0.605 & 0.130 & 20.16 \\
\hline PsNAC14 vs. $M D 08 G 1222300$ & 0.085 & 0.400 & 0.211 & 13.33 \\
\hline PsNAC14 vs. $M D 15 G 1415700$ & 0.091 & 0.398 & 0.228 & 13.26 \\
\hline PsNAC16 vs. $M D 02 G 1243800$ & 0.417 & 1.551 & 0.269 & 51.70 \\
\hline PsNAC17 vs. $M D 02 G 1243200$ & 0.135 & 0.424 & 0.318 & 14.15 \\
\hline PsNAC17 vs. $M D 07 G 1073100$ & 0.688 & 1.907 & 0.361 & 63.58 \\
\hline PsNAC18 vs. $M D 01 G 1088100$ & 0.107 & 0.556 & 0.193 & 18.54 \\
\hline PsNAC18 vs. $M D 07 G 1158300$ & 0.073 & 0.469 & 0.155 & 15.64 \\
\hline PsNAC18 vs. $M D 14 G 1137900$ & 0.308 & 1.432 & 0.215 & 47.73 \\
\hline PsNAC20 vs. $M D 01 G 1092200$ & 0.363 & 1.075 & 0.338 & 35.82 \\
\hline PsNAC21 vs. $M D 01 G 1092200$ & 0.296 & 0.715 & 0.414 & 23.82 \\
\hline PsNAC21 vs. $M D 07 G 1162700$ & 0.461 & 0.807 & 0.571 & 26.89 \\
\hline PsNAC22 vs. $M D 01 G 1092900$ & 0.424 & 0.888 & 0.478 & 29.58 \\
\hline PsNAC23 vs. $M D 01 G 1093000$ & 0.538 & 0.753 & 0.714 & 25.11 \\
\hline PsNAC25 vs. $M D 07 G 1162800$ & 0.363 & 0.407 & 0.893 & 13.56 \\
\hline PsNAC27 vs. $M D 01 G 1092600$ & 0.410 & 0.816 & 0.503 & 27.19 \\
\hline PsNAC28 vs. $M D 01 G 1093500$ & 0.391 & 0.670 & 0.584 & 22.34 \\
\hline PsNAC29 vs. $M D 01 G 1093900$ & 0.219 & 0.337 & 0.651 & 11.23 \\
\hline PsNAC30 vs. $M D 01 G 1093800$ & 0.249 & 0.590 & 0.422 & 19.66 \\
\hline PsNAC31 vs. $M D 01 G 1094700$ & 0.319 & 0.490 & 0.650 & 16.33 \\
\hline PsNAC31 vs. $M D 07 G 1163400$ & 0.389 & 0.514 & 0.757 & 17.14 \\
\hline PsNAC32 vs. $M D 01 G 1094700$ & 0.373 & 0.546 & 0.683 & 18.20 \\
\hline PsNAC36 vs. $M D 07 G 1254800$ & 0.018 & 0.345 & 0.051 & 11.50 \\
\hline PsNAC37 vs. MD01G1229900 & 0.141 & 0.485 & 0.290 & 16.18 \\
\hline PsNAC37 vs. $M D 06 G 1124800$ & 0.422 & 2.557 & 0.165 & 85.24 \\
\hline PsNAC37 vs. $M D 07 G 1301800$ & 0.134 & 0.481 & 0.278 & 16.02 \\
\hline PsNAC38 vs. $M D 06 G 1135700$ & 0.560 & 1.824 & 0.307 & 60.78 \\
\hline PsNAC38 vs. $M D 09 G 1006400$ & 0.200 & 0.478 & 0.419 & 15.94 \\
\hline PsNAC 38 vs. $M D 14 G 1150900$ & 0.525 & 1.759 & 0.299 & 58.63 \\
\hline PsNAC38 vs. $M D 16 G 1125800$ & 0.491 & 2.598 & 0.189 & 86.59 \\
\hline PsNAC38 vs. $M D 17 G 1010300$ & 0.208 & 0.485 & 0.429 & 16.16 \\
\hline PsNAC39 vs. $M D 09 G 1023600$ & 0.096 & 0.235 & 0.408 & 7.83 \\
\hline PsNAC39 vs. $M D 17 G 1023100$ & 0.128 & 0.319 & 0.400 & 10.62 \\
\hline PsNAC40 vs. $M D 09 G 1050400$ & 0.069 & 0.495 & 0.140 & 16.49 \\
\hline PsNAC40 vs. $M D 13 G 1072500$ & 0.341 & 1.769 & 0.193 & 58.96 \\
\hline PsNAC40 vs. $M D 16 G 1073300$ & 0.348 & 1.450 & 0.240 & 48.34 \\
\hline PsNAC40 vs. $M D 17 G 1050600$ & 0.084 & 0.469 & 0.178 & 15.63 \\
\hline PsNAC41 vs. $M D 09 G 1053700$ & 0.082 & 0.562 & 0.145 & 18.74 \\
\hline
\end{tabular}


Supplemental Table 5. (Continued)

\begin{tabular}{|c|c|c|c|c|}
\hline Duplication gene pairs & $\mathrm{Ka}$ & $\mathrm{Ks}$ & $\mathrm{Ka} / \mathrm{Ks}$ & $\begin{array}{l}\text { Divergence time } \\
(\text { Mya })^{\mathrm{z}}\end{array}$ \\
\hline PsNAC41 vs. $M D 17 G 1051600$ & 0.082 & 0.591 & 0.140 & 19.70 \\
\hline PsNAC42 vs. $M D 09 G 1147500$ & 0.117 & 0.311 & 0.376 & 10.35 \\
\hline PsNAC42 vs. $M D 17 G 1134400$ & 0.100 & 0.282 & 0.356 & 9.39 \\
\hline PsNAC46 vs. $M D 15 G 1136600$ & 0.083 & 0.593 & 0.140 & 19.76 \\
\hline PsNAC47 vs. $M D 03 G 1172900$ & 0.257 & 0.680 & 0.378 & 22.67 \\
\hline PsNAC47 vs. $M D 11 G 1190900$ & 0.279 & 0.704 & 0.396 & 23.46 \\
\hline PsNAC50 vs. $M D 03 G 1222600$ & 0.076 & 0.581 & 0.131 & 19.37 \\
\hline PsNAC50 vs. $M D 05 G 1321800$ & 0.147 & 1.474 & 0.100 & 49.13 \\
\hline PsNAC50 vs. $M D 10 G 1299900$ & 0.292 & 2.215 & 0.132 & 73.84 \\
\hline PsNAC50 vs. $M D 11 G 1239900$ & 0.065 & 0.641 & 0.102 & 21.36 \\
\hline PsNAC51 vs. $M D 03 G 1222700$ & 0.106 & 0.627 & 0.169 & 20.90 \\
\hline PsNAC52 vs. $M D 11 G 1253500$ & 0.069 & 0.624 & 0.110 & 20.79 \\
\hline PsNAC54 vs. $M D 03 G 1255100$ & 0.255 & 0.488 & 0.523 & 16.25 \\
\hline PsNAC55 vs. $M D 05 G 1221100$ & 0.087 & 0.489 & 0.178 & 16.29 \\
\hline PsNAC56 vs. $M D 05 G 1239300$ & 0.045 & 0.341 & 0.133 & 11.38 \\
\hline PsNAC56 vs. MD10G1217500 & 0.043 & 0.325 & 0.132 & 10.85 \\
\hline PsNAC57 vs. $M D 10 G 1220600$ & 0.047 & 0.479 & 0.098 & 15.95 \\
\hline PsNAC58 vs. $M D 05 G 1258300$ & 0.112 & 0.428 & 0.262 & 14.27 \\
\hline PsNAC58 vs. $M D 10 G 1238200$ & 0.102 & 0.494 & 0.207 & 16.46 \\
\hline PsNAC59 vs. $M D 05 G 1309700$ & 0.033 & 0.503 & 0.065 & 16.76 \\
\hline PsNAC59 vs. $M D 10 G 1288300$ & 0.036 & 0.525 & 0.068 & 17.50 \\
\hline PsNAC60 vs. $M D 05 G 1321800$ & 0.081 & 0.605 & 0.133 & 20.16 \\
\hline PsNAC60 vs. $M D 10 G 1299900$ & 0.123 & 0.561 & 0.220 & 18.70 \\
\hline PsNAC61 vs. $M D 07 G 1254700$ & 0.054 & 0.411 & 0.132 & 13.69 \\
\hline PsNAC61 vs. $M D 11 G 1195600$ & 0.038 & 0.536 & 0.072 & 17.85 \\
\hline PsNAC62 vs. $M D 06 G 1004900$ & 0.161 & 0.644 & 0.250 & 21.46 \\
\hline PsNAC64 vs. $M D 06 G 1121400$ & 0.042 & 0.481 & 0.087 & 16.02 \\
\hline PsNAC64 vs. $M D 07 G 1158300$ & 0.314 & $\mathrm{NaN}$ & $\mathrm{NaN}$ & $\mathrm{NaN}$ \\
\hline PsNAC64 vs. $M D 14 G 1137900$ & 0.051 & 0.440 & 0.115 & 14.67 \\
\hline PsNAC65 vs. $M D 06 G 1124800$ & 0.128 & 0.393 & 0.327 & 13.09 \\
\hline PsNAC66 vs. $M D 06 G 1135700$ & 0.087 & 0.613 & 0.142 & 20.43 \\
\hline PsNAC66 vs. MD14G1150900 & 0.117 & 0.513 & 0.228 & 17.09 \\
\hline PsNAC66 vs. $M D 16 G 1125800$ & 0.299 & 2.267 & 0.132 & 75.58 \\
\hline PsNAC67 vs. $M D 06 G 1196100$ & 0.079 & 0.522 & 0.151 & 17.42 \\
\hline PsNAC67 vs. $M D 09 G 1053700$ & 0.269 & 1.400 & 0.192 & 46.67 \\
\hline PsNAC67 vs. $M D 14 G 1203000$ & 0.085 & 0.447 & 0.191 & 14.91 \\
\hline PsNAC68 vs. $M D 14 G 1210000$ & 0.219 & 0.539 & 0.406 & 17.96 \\
\hline PsNAC69 vs. $M D 06 G 1216500$ & 0.124 & 0.531 & 0.233 & 17.71 \\
\hline PsNAC69 vs. $M D 14 G 1226900$ & 0.133 & 0.510 & 0.261 & 17.02 \\
\hline PsNAC70 vs. $M D 06 G 1236800$ & 0.185 & 0.764 & 0.241 & 25.48 \\
\hline PsNAC70 vs. $M D 14 G 1243700$ & 0.107 & 0.494 & 0.216 & 16.48 \\
\hline PsNAC71 vs. $M D 11 G 1074200$ & 0.412 & 1.076 & 0.383 & 35.88 \\
\hline PsNAC75 vs. $M D 03 G 1115100$ & 0.134 & 0.428 & 0.314 & 14.26 \\
\hline PsNAC75 vs. $M D 11 G 1133000$ & 0.132 & 0.369 & 0.357 & 12.28 \\
\hline PsNAC76 vs. $M D 11 G 1138500$ & 0.451 & 0.981 & 0.460 & 32.69 \\
\hline PsNAC79 vs. $M D 03 G 1130400$ & 0.554 & 1.604 & 0.345 & 53.45 \\
\hline PsNAC81 vs. $M D 03 G 1148500$ & 0.284 & 0.682 & 0.417 & 22.73 \\
\hline PsNAC81 vs. $M D 11 G 1167900$ & 0.219 & 0.588 & 0.372 & 19.60 \\
\hline PsNAC81 vs. $M D 12 G 1030200$ & 0.627 & 1.525 & 0.411 & 50.84 \\
\hline PsNAC81 vs. $M D 14 G 1031000$ & 0.695 & 2.054 & 0.339 & 68.47 \\
\hline PsNAC82 vs. $M D 03 G 1150800$ & 0.140 & 0.396 & 0.354 & 13.21 \\
\hline PsNAC82 vs. $M D 11 G 1170300$ & 0.113 & 0.299 & 0.379 & 9.95 \\
\hline PsNAC83 vs. $M D 03 G 1115100$ & 0.479 & 1.810 & 0.265 & 60.34 \\
\hline PsNAC83 vs. $M D 04 G 1100400$ & 0.082 & 0.479 & 0.172 & 15.97 \\
\hline PsNAC83 vs. $M D 11 G 1133000$ & 0.464 & 1.500 & 0.309 & 49.99 \\
\hline PsNAC84 vs. $M D 02 G 1068700$ & 0.041 & 0.279 & 0.148 & 9.29 \\
\hline
\end{tabular}


Supplemental Table 5. (Continued)

\begin{tabular}{|c|c|c|c|c|}
\hline Duplication gene pairs & $\mathrm{Ka}$ & Ks & $\mathrm{Ka} / \mathrm{Ks}$ & $\begin{array}{l}\text { Divergence time } \\
(\text { Mya })^{\mathrm{z}}\end{array}$ \\
\hline PsNAC84 vs. $M D 15 G 1200200$ & 0.045 & 0.315 & 0.143 & 10.49 \\
\hline PsNAC85 vs. $M D 02 G 1134500$ & 0.044 & 0.359 & 0.123 & 11.95 \\
\hline PsNAC85 vs. $M D 15 G 1247800$ & 0.038 & 0.421 & 0.091 & 14.04 \\
\hline PsNAC86 vs. $M D 12 G 1030200$ & 0.200 & 0.544 & 0.368 & 18.15 \\
\hline PsNAC86 vs. $M D 14 G 1030700$ & 0.237 & 0.535 & 0.444 & 17.82 \\
\hline PsNAC88 vs. $M D 14 G 1069400$ & 0.364 & 0.608 & 0.598 & 20.28 \\
\hline PsNAC90 vs. $M D 14 G 1071200$ & 0.421 & 0.646 & 0.653 & 21.52 \\
\hline PsNAC92 vs. $M D 12 G 1000800$ & 0.102 & 0.385 & 0.264 & 12.85 \\
\hline PsNAC92 vs. $M D 14 G 1001000$ & 0.100 & 0.417 & 0.240 & 13.89 \\
\hline PsNAC93 vs. $M D 05 G 1195300$ & 0.064 & 0.339 & 0.188 & 11.29 \\
\hline PsNAC94 vs. $M D 05 G 1130500$ & 0.074 & 0.370 & 0.201 & 12.34 \\
\hline PsNAC94 vs. $M D 10 G 1133400$ & 0.072 & 0.335 & 0.215 & 11.16 \\
\hline PsNAC97 vs. $M D 05 G 1042100$ & 0.100 & 0.385 & 0.260 & 12.83 \\
\hline PsNAC97 vs. $M D 10 G 1048600$ & 0.075 & 0.346 & 0.218 & 11.55 \\
\hline PsNAC98 vs. MD05G1037700 & 0.109 & 0.289 & 0.376 & 9.64 \\
\hline PsNAC98 vs. $M D 10 G 1041500$ & 0.108 & 0.272 & 0.397 & 9.08 \\
\hline PsNAC99 vs. MD05G1029800 & 0.378 & 0.762 & 0.496 & 25.41 \\
\hline PsNAC99 vs. MD10G1030400 & 0.250 & 0.460 & 0.544 & 15.33 \\
\hline
\end{tabular}

${ }^{\mathrm{z}}$ Million years ago.

Supplemental Table 6. Estimated non-synonymous (Ka) to synonymous (Ks) substitution ratios and divergence times of the syntenic gene pairs of NAC between siberian apricot and peach.

\begin{tabular}{|c|c|c|c|c|}
\hline Duplication gene pairs & $\mathrm{Ka}$ & Ks & $\mathrm{Ka} / \mathrm{Ks}$ & $\begin{array}{l}\text { Divergence time } \\
(\mathrm{Mya})^{\mathrm{z}}\end{array}$ \\
\hline PsNAC2 vs. Prupe.1G106100.1 & 0.020 & 0.054 & 0.371 & 1.80 \\
\hline PsNAC4 vs. Prupe. $1 G 220400.1$ & 0.012 & 0.023 & 0.500 & 0.78 \\
\hline PsNAC4 vs. Prupe. $3 G 310600.1$ & 0.465 & 1.891 & 0.246 & 63.03 \\
\hline PsNAC4 vs. Prupe. $5 G 146100.1$ & 0.271 & 1.222 & 0.222 & 40.75 \\
\hline PsNAC5 vs. Prupe. $1 G 274300.1$ & 0.018 & 0.055 & 0.324 & 1.85 \\
\hline PsNAC5 vs. Prupe. $3 G 268400.1$ & 0.320 & 1.499 & 0.213 & 49.96 \\
\hline PsNAC6 vs. Prupe.1G277500.1 & 0.007 & 0.087 & 0.085 & 2.89 \\
\hline PsNAC7 vs. Prupe. $1 G 284800.1$ & 0.162 & 0.208 & 0.777 & 6.94 \\
\hline PsNAC9 vs. Prupe. $1 G 305300.1$ & 0.014 & 0.025 & 0.573 & 0.83 \\
\hline PsNAC10 vs. Prupe.1G383200.1 & 0.017 & 0.045 & 0.382 & 1.48 \\
\hline PsNAC11 vs. Prupe. $1 G 432600.2$ & 0.108 & 0.138 & 0.784 & 4.60 \\
\hline PsNAC12 vs. Prupe. $1 G 454900.2$ & 0.004 & 0.026 & 0.145 & 0.87 \\
\hline PsNAC13 vs. Prupe.1G493100.1 & 0.016 & 0.074 & 0.218 & 2.48 \\
\hline PsNAC14 vs. Prupe.1G560100.1 & 0.019 & 0.086 & 0.220 & 2.88 \\
\hline PsNAC16 vs. Prupe. $2 G 091300.1$ & 0.012 & 0.023 & 0.532 & 0.78 \\
\hline PsNAC18 vs. Prupe. 2 G196600.1 & 0.009 & 0.036 & 0.241 & 1.19 \\
\hline PsNAC18 vs. Prupe. 5 G131900.1 & 0.321 & 1.976 & 0.162 & 65.87 \\
\hline PsNAC20 vs. Prupe. $2 G 201200.1$ & 0.105 & 0.116 & 0.905 & 3.88 \\
\hline PsNAC21 vs. Prupe. $2 G 201200.1$ & 0.175 & 0.223 & 0.786 & 7.43 \\
\hline PsNAC25 vs. Prupe. $2 G 202200.1$ & 0.276 & 0.277 & 0.999 & 9.22 \\
\hline PsNAC26 vs. Prupe. $2 G 201300.1$ & 0.266 & 0.316 & 0.842 & 10.55 \\
\hline PsNAC28 vs. Prupe. $2 G 201800.1$ & 0.241 & 0.378 & 0.638 & 12.59 \\
\hline PsNAC28 vs. Prupe. $2 G 202600.1$ & 0.477 & 0.789 & 0.604 & 26.30 \\
\hline PsNAC29 vs. Prupe. $2 G 202500.1$ & 0.021 & 0.025 & 0.860 & 0.83 \\
\hline PsNAC31 vs. Prupe. $2 G 202500.5$ & 0.531 & 1.214 & 0.437 & 40.48 \\
\hline PsNAC31 vs. Prupe. $2 G 202800.1$ & 0.079 & 0.130 & 0.612 & 4.32 \\
\hline PsNAC32 vs. Prupe. $2 G 202800.1$ & 0.193 & 0.212 & 0.912 & 7.05 \\
\hline PsNAC33 vs. Prupe. $2 G 204700.1$ & 0.157 & 0.256 & 0.613 & 8.53 \\
\hline PsNAC36 vs. Prupe. $2 G 278100.1$ & 0.040 & 0.089 & 0.449 & 2.96 \\
\hline PsNAC37 vs. Prupe. 2 G320600.1 & 0.026 & 0.068 & 0.378 & 2.27 \\
\hline PsNAC37 vs. Prupe. 5 G135400.1 & 0.437 & 3.324 & 0.131 & 110.81 \\
\hline
\end{tabular}


Supplemental Table 6. (Continued)

\begin{tabular}{|c|c|c|c|c|}
\hline Duplication gene pairs & $\mathrm{Ka}$ & Ks & $\mathrm{Ka} / \mathrm{Ks}$ & $\begin{array}{l}\text { Divergence time } \\
(\mathrm{Mya})^{\mathrm{z}}\end{array}$ \\
\hline PsNAC38 vs. Prupe.1G220400.1 & 0.484 & 1.449 & 0.334 & 48.32 \\
\hline PsNAC38 vs. Prupe. $3 G 310600.1$ & 0.020 & 0.026 & 0.791 & 0.85 \\
\hline PsNAC38 vs. Prupe. $5 G 146100.1$ & 0.635 & 3.093 & 0.205 & 103.09 \\
\hline PsNAC39 vs. Prupe.3G293200.2 & 0.009 & 0.036 & 0.247 & 1.19 \\
\hline PsNAC40 vs. Prupe. $1 G 274300.1$ & 0.312 & 1.226 & 0.254 & 40.88 \\
\hline PsNAC40 vs. Prupe. $3 G 268400.1$ & 0.010 & 0.028 & 0.348 & 0.93 \\
\hline PsNAC41 vs. Prupe. $3 G 264600.1$ & 0.012 & 0.050 & 0.243 & 1.67 \\
\hline PsNAC42 vs. Prupe. $3 G 178000.1$ & 0.014 & 0.064 & 0.220 & 2.13 \\
\hline PsNAC43 vs. Prupe. $3 G 111400.1$ & 0.036 & 0.027 & 1.320 & 0.91 \\
\hline PsNAC45 vs. Prupe. $3 G 065000.1$ & 0.035 & 0.055 & 0.641 & 1.83 \\
\hline PsNAC46 vs. Prupe.4G279600.1 & 0.009 & 0.044 & 0.198 & 1.47 \\
\hline PsNAC47 vs. Prupe.4G253600.1 & 0.048 & 0.071 & 0.679 & 2.36 \\
\hline PsNAC48 vs. Prupe. $4 G 230600.1$ & 0.505 & 1.215 & 0.415 & 40.50 \\
\hline PsNAC50 vs. Prupe. $4 G 187100.1$ & 0.011 & 0.059 & 0.183 & 1.96 \\
\hline PsNAC51 vs. Prupe.4G186800.1 & 0.015 & 0.065 & 0.233 & 2.15 \\
\hline PsNAC52 vs. Prupe.4G175800.1 & 0.005 & 0.050 & 0.091 & 1.66 \\
\hline PsNAC54 vs. Prupe.4G151900.1 & 0.017 & 0.074 & 0.232 & 2.46 \\
\hline PsNAC55 vs. Prupe.4G138500.1 & 0.009 & 0.056 & 0.165 & 1.87 \\
\hline PsNAC56 vs. Prupe.4G126800.1 & 0.012 & 0.035 & 0.352 & 1.16 \\
\hline PsNAC57 vs. Prupe.4G123700.2 & 0.031 & 0.093 & 0.335 & 3.11 \\
\hline PsNAC58 vs. Prupe. $4 G 106100.1$ & 0.022 & 0.041 & 0.535 & 1.38 \\
\hline PsNAC59 vs. Prupe.4G053300.1 & 0.003 & 0.033 & 0.082 & 1.11 \\
\hline PsNAC60 vs. Prupe.4G040900.1 & 0.012 & 0.033 & 0.367 & 1.09 \\
\hline PsNAC61 vs. Prupe.2G278100.1 & 0.000 & 0.047 & 0.000 & 1.56 \\
\hline PsNAC62 vs. Prupe.5G006200.1 & 0.015 & 0.035 & 0.422 & 1.18 \\
\hline PsNAC63 vs. Prupe.5G076100.1 & 0.005 & 0.035 & 0.134 & 1.17 \\
\hline PsNAC64 vs. Prupe.2G196600.1 & 0.301 & 2.465 & 0.122 & 82.18 \\
\hline PsNAC64 vs. Prupe.5G131900.1 & 0.000 & 0.032 & 0.000 & 1.06 \\
\hline PsNAC65 vs. Prupe.2G320600.1 & 0.513 & 2.637 & 0.194 & 87.90 \\
\hline PsNAC65 vs. Prupe.5G135400.1 & 0.017 & 0.046 & 0.368 & 1.53 \\
\hline PsNAC66 vs. Prupe.1G220400.1 & 0.266 & 1.092 & 0.244 & 36.41 \\
\hline PsNAC66 vs. Prupe.3G310600.1 & 0.616 & 3.010 & 0.205 & 100.32 \\
\hline PsNAC66 vs. Prupe.5G146100.1 & 0.010 & 0.061 & 0.160 & 2.05 \\
\hline PsNAC67 vs. Prupe. $3 G 264600.1$ & 0.227 & 1.330 & 0.170 & 44.32 \\
\hline PsNAC67 vs. Prupe.5G196000.1 & 0.018 & 0.042 & 0.428 & 1.41 \\
\hline PsNAC68 vs. Prupe.5G203200.1 & 0.081 & 0.093 & 0.864 & 3.11 \\
\hline PsNAC69 vs. Prupe.5G221600.1 & 0.007 & 0.030 & 0.240 & 1.01 \\
\hline PsNAC70 vs. Prupe.5G241300.1 & 0.002 & 0.053 & 0.031 & 1.77 \\
\hline PsNAC71 vs. Prupe.6G057300.1 & 0.040 & 0.041 & 0.970 & 1.36 \\
\hline PsNAC72 vs. Prupe.6G092100.1 & 0.029 & 0.041 & 0.689 & 1.38 \\
\hline PsNAC73 vs. Prupe.6G098300.1 & 0.204 & 0.423 & 0.482 & 14.09 \\
\hline PsNAC75 vs. Prupe.6G098600.1 & 0.022 & 0.053 & 0.404 & 1.78 \\
\hline PsNAC75 vs. Prupe. $6 G 238600.1$ & 0.434 & 1.706 & 0.254 & 56.88 \\
\hline PsNAC76 vs. Prupe.6G103600.1 & 0.089 & 0.228 & 0.391 & 7.59 \\
\hline PsNAC79 vs. Prupe.6G112000.1 & 0.021 & 0.023 & 0.922 & 0.76 \\
\hline PsNAC81 vs. Prupe. $6 G 134400.1$ & 0.151 & 0.180 & 0.837 & 6.00 \\
\hline PsNAC81 vs. Prupe.7G102000.1 & 0.540 & 1.521 & 0.355 & 50.71 \\
\hline PsNAC82 vs. Prupe.6G138100.1 & 0.010 & 0.035 & 0.291 & 1.15 \\
\hline PsNAC83 vs. Prupe.6G238600.1 & 0.014 & 0.047 & 0.297 & 1.56 \\
\hline PsNAC83 vs. Prupe.6G098600.1 & 0.455 & 1.747 & 0.260 & 58.23 \\
\hline PsNAC84 vs. Prupe. $7 G 217000.2$ & 0.001 & 0.033 & 0.026 & 1.11 \\
\hline PsNAC85 vs. Prupe. $7 G 166300.1$ & 0.000 & 0.039 & 0.000 & 1.30 \\
\hline PsNAC86 vs. Prupe.6G134400.1 & 0.406 & 2.753 & 0.148 & 91.77 \\
\hline PsNAC86 vs. Prupe.7G102000.1 & 0.093 & 0.116 & 0.805 & 3.85 \\
\hline PsNAC87 vs. Prupe. $7 G 092400.2$ & 0.028 & 0.084 & 0.328 & 2.81 \\
\hline
\end{tabular}


Supplemental Table 6. (Continued)

\begin{tabular}{|c|c|c|c|c|}
\hline Duplication gene pairs & $\mathrm{Ka}$ & Ks & $\mathrm{Ka} / \mathrm{Ks}$ & $\begin{array}{c}\text { Divergence time } \\
(\mathrm{Mya})^{\mathrm{z}}\end{array}$ \\
\hline PsNAC88 vs. Prupe. $7 G 061900.1$ & 0.718 & 1.849 & 0.388 & 61.63 \\
\hline PsNAC88 vs. Prupe. $7 G 064900.1$ & 0.502 & 0.793 & 0.633 & 26.44 \\
\hline PsNAC90 vs. Prupe. $7 G 065000.1$ & 0.746 & $\mathrm{NaN}$ & $\mathrm{NaN}$ & $\mathrm{NaN}$ \\
\hline PsNAC90 vs. Prupe.7G061900.1 & 0.038 & 0.080 & 0.478 & 2.66 \\
\hline PsNAC91 vs. Prupe.7G013900.1 & 0.096 & 0.098 & 0.974 & 3.27 \\
\hline PsNAC92 vs. Prupe.7G001200.1 & 0.010 & 0.052 & 0.194 & 1.75 \\
\hline PsNAC93 vs. Prupe. $8 G 221900.1$ & 0.018 & 0.058 & 0.314 & 1.93 \\
\hline PsNAC94 vs. Prupe. $8 G 174800.1$ & 0.016 & 0.043 & 0.375 & 1.45 \\
\hline PsNAC95 vs. Prupe. $8 G 097100.1$ & 0.026 & 0.049 & 0.534 & 1.64 \\
\hline PsNAC95 vs. Prupe.8G095200.1 & 0.042 & 0.111 & 0.374 & 3.71 \\
\hline PsNAC96 vs. Prupe. $8 G 074800.1$ & 0.084 & 0.138 & 0.608 & 4.61 \\
\hline PsNAC97 vs. Prupe. $8 G 058100.1$ & 0.010 & 0.043 & 0.224 & 1.44 \\
\hline PsNAC98 vs. Prupe. $8 G 045800.1$ & 0.012 & 0.034 & 0.354 & 1.13 \\
\hline PsNAC99 vs. Prupe. $8 G 035300.1$ & 0.267 & 0.476 & 0.560 & 15.87 \\
\hline PsNAC102 vs. Prupe. $8 G 002500.1$ & 0.006 & 0.067 & 0.084 & 2.24 \\
\hline
\end{tabular}

${ }^{\mathrm{z}}$ Million years ago.

Supplemental Table 7. Estimated non-synonymous (Ka) to synonymous (Ks) substitution ratios and divergence times of the syntenic gene pairs of NAC between siberian apricot and japanese apricot.

\begin{tabular}{|c|c|c|c|c|}
\hline Duplication gene pairs & $\mathrm{Ka}$ & $\mathrm{Ks}$ & $\mathrm{Ka} / \mathrm{Ks}$ & $\begin{array}{c}\text { Divergence time } \\
(\mathrm{Mya})^{\mathrm{z}}\end{array}$ \\
\hline$P s N A C 2$ vs. $P m 008481$ & $\mathrm{NaN}$ & $\mathrm{NaN}$ & $\mathrm{NaN}$ & $\mathrm{NaN}$ \\
\hline PsNAC4 vs. Pm007070 & 0.005 & 0.018 & 0.284 & 0.59 \\
\hline PsNAC4 vs. Pm016114 & 0.482 & 1.696 & 0.284 & 56.55 \\
\hline PsNAC4 vs. $P m 024558$ & 0.271 & 1.219 & 0.222 & 40.63 \\
\hline PsNAC5 vs. Pm006506 & 0.004 & 0.019 & 0.234 & 0.63 \\
\hline PsNAC5 vs. Pm015876 & 0.330 & 1.360 & 0.243 & 45.34 \\
\hline PsNAC6 vs. Pm006470 & 0.004 & 0.017 & 0.267 & 0.55 \\
\hline PsNAC7 vs. Pm006394 & 0.248 & 0.351 & 0.706 & 11.71 \\
\hline PsNAC9 vs. $P m 029010$ & 0.006 & 0.019 & 0.318 & 0.62 \\
\hline PsNAC10 vs. Pm005783 & 0.009 & 0.028 & 0.322 & 0.94 \\
\hline PsNAC11 vs. Pm005306 & 0.103 & 0.138 & 0.743 & 4.61 \\
\hline PsNAC12 vs. Pm005091 & 0.003 & 0.022 & 0.116 & 0.72 \\
\hline PsNAC13 vs. Pm004758 & 0.009 & 0.121 & 0.073 & 4.02 \\
\hline PsNAC14 vs. Pm004078 & 0.006 & 0.028 & 0.226 & 0.95 \\
\hline PsNAC18 vs. Pm018292 & 0.005 & 0.019 & 0.275 & 0.65 \\
\hline$P s N A C 21$ vs. $P m 028718$ & 0.360 & 0.487 & 0.739 & 16.22 \\
\hline$P s N A C 30$ vs. Pm028713 & 0.023 & 0.042 & 0.540 & 1.41 \\
\hline$P s N A C 32$ vs. $P m 028710$ & 0.014 & 0.015 & 0.912 & 0.50 \\
\hline PsNAC33 vs. Pm018442 & 0.162 & 0.208 & 0.781 & 6.92 \\
\hline$P s N A C 36$ vs. $P m 019488$ & 0.049 & 0.093 & 0.531 & 3.09 \\
\hline PsNAC37 vs. Pm019659 & 0.012 & 0.036 & 0.348 & 1.19 \\
\hline$P s N A C 38$ vs. $P m 007070$ & 0.454 & 2.417 & 0.188 & 80.58 \\
\hline PsNAC38 vs. Pm016114 & 0.007 & 0.019 & 0.380 & 0.64 \\
\hline PsNAC38 vs. Pm024558 & 0.640 & 2.461 & 0.260 & 82.04 \\
\hline PsNAC40 vs. Pm006506 & 0.306 & 1.178 & 0.260 & 39.27 \\
\hline$P s N A C 40$ vs. Pm015876 & 0.010 & 0.016 & 0.614 & 0.53 \\
\hline PsNAC41 vs. Pm015914 & 0.010 & 0.013 & 0.729 & 0.45 \\
\hline PsNAC42 vs. Pm014764 & 0.006 & 0.010 & 0.546 & 0.34 \\
\hline PsNAC45 vs. Pm013492 & 0.011 & 0.013 & 0.886 & 0.42 \\
\hline$P s N A C 46$ vs. Pm012630 & 0.001 & 0.011 & 0.135 & 0.36 \\
\hline
\end{tabular}


Supplemental Table 7. (Continued)

\begin{tabular}{|c|c|c|c|c|}
\hline Duplication gene pairs & $\mathrm{Ka}$ & $\mathrm{Ks}$ & $\mathrm{Ka} / \mathrm{Ks}$ & $\begin{array}{c}\text { Divergence time } \\
(\mathrm{Mya})^{\mathrm{z}}\end{array}$ \\
\hline PsNAC47 vs. Pm012336 & 0.038 & 0.058 & 0.645 & 1.94 \\
\hline PsNAC48 vs. Pm011991 & 0.566 & 1.600 & 0.354 & 53.33 \\
\hline$P s N A C 50$ vs. $P m 009954$ & 0.451 & 1.626 & 0.277 & 54.21 \\
\hline PsNAC50 vs. Pm011604 & 0.009 & 0.016 & 0.583 & 0.53 \\
\hline PsNAC51 vs. Pm009955 & 0.296 & 1.782 & 0.166 & 59.40 \\
\hline$P s N A C 51$ vs. $P m 011603$ & 0.007 & 0.012 & 0.605 & 0.41 \\
\hline PsNAC52 vs. Pm011397 & 0.000 & 0.030 & 0.000 & 1.00 \\
\hline PsNAC54 vs. Pm011146 & 0.005 & 0.018 & 0.285 & 0.59 \\
\hline PsNAC55 vs. Pm011037 & 0.008 & 0.036 & 0.224 & 1.18 \\
\hline PsNAC56 vs. $P m 010926$ & 0.005 & 0.017 & 0.285 & 0.57 \\
\hline PsNAC57 vs. Pm010886 & 0.016 & 0.058 & 0.282 & 1.94 \\
\hline$P s N A C 58$ vs. $P m 010673$ & 0.003 & 0.015 & 0.184 & 0.51 \\
\hline PsNAC59 vs. Pm010104 & 0.000 & 0.024 & 0.000 & 0.79 \\
\hline$P s N A C 60$ vs. Pm009954 & 0.011 & 0.018 & 0.612 & 0.59 \\
\hline PsNAC61 vs. Pm019488 & 0.010 & 0.047 & 0.213 & 1.56 \\
\hline PsNAC62 vs. Pm022916 & 0.002 & 0.032 & 0.062 & 1.06 \\
\hline PsNAC63 vs. Pm023559 & 0.012 & 0.042 & 0.297 & 1.39 \\
\hline PsNAC64 vs. Pm018292 & 0.325 & 2.033 & 0.160 & 67.77 \\
\hline PsNAC64 vs. Pm024260 & 0.001 & 0.024 & 0.045 & 0.79 \\
\hline PsNAC65 vs. Pm019659 & 0.529 & 3.259 & 0.162 & 108.65 \\
\hline PsNAC66 vs. Pm007070 & 0.271 & 1.263 & 0.215 & 42.11 \\
\hline PsNAC66 vs. Pm016114 & 0.627 & 2.637 & 0.238 & 87.91 \\
\hline$P s N A C 66$ vs. $P m 024558$ & 0.011 & 0.054 & 0.205 & 1.80 \\
\hline PsNAC67 vs. Pm015914 & 0.233 & 1.235 & 0.189 & 41.16 \\
\hline PsNAC67 vs. Pm024887 & 0.011 & 0.021 & 0.521 & 0.69 \\
\hline PsNAC68 vs. Pm024976 & 0.086 & 0.091 & 0.950 & 3.03 \\
\hline PsNAC69 vs. Pm025184 & 0.203 & 0.389 & 0.521 & 12.97 \\
\hline$P s N A C 70$ vs. $P m 025250$ & 0.069 & 0.136 & 0.506 & 4.54 \\
\hline PsNAC71 vs. $P m 000566$ & 0.063 & 0.107 & 0.589 & 3.57 \\
\hline PsNAC72 vs. Pm000962 & 0.062 & 0.119 & 0.524 & 3.97 \\
\hline$P s N A C 73$ vs. $P m 000998$ & 0.019 & 0.037 & 0.529 & 1.22 \\
\hline$P s N A C 75$ vs. $P m 001001$ & 0.010 & 0.020 & 0.505 & 0.66 \\
\hline PsNAC76 vs. Pm001039 & 0.078 & 0.131 & 0.596 & 4.37 \\
\hline PsNAC78 vs. $P m 001136$ & 0.073 & 0.114 & 0.640 & 3.79 \\
\hline PsNAC79 vs. $P m 001146$ & 0.031 & 0.031 & 0.991 & 1.03 \\
\hline PsNAC80 vs. Pm001157 & 0.025 & 0.027 & 0.922 & 0.91 \\
\hline PsNAC81 vs. $P m 001403$ & 0.136 & 0.167 & 0.815 & 5.56 \\
\hline PsNAC81 vs. $P m 028056$ & 0.571 & 1.668 & 0.342 & 55.60 \\
\hline PsNAC82 vs. $P m 001467$ & 0.007 & 0.017 & 0.386 & 0.57 \\
\hline PsNAC83 vs. $P m 001001$ & 0.520 & 1.618 & 0.321 & 53.92 \\
\hline PsNAC83 vs. Pm030099 & 0.013 & 0.024 & 0.525 & 0.80 \\
\hline PsNAC84 vs. Pm027280 & 0.002 & 0.033 & 0.052 & 1.11 \\
\hline$P s N A C 85$ vs. $P m 026735$ & 0.003 & 0.029 & 0.087 & 0.97 \\
\hline PsNAC86 vs. Pm001403 & 0.402 & 2.303 & 0.175 & 76.77 \\
\hline PsNAC86 vs. Pm028056 & 0.107 & 0.113 & 0.945 & 3.78 \\
\hline PsNAC87 vs. Pm026045 & 0.012 & 0.026 & 0.480 & 0.86 \\
\hline PsNAC88 vs. $P m 025886$ & 0.017 & 0.019 & 0.927 & 0.62 \\
\hline PsNAC91 vs. $P m 028678$ & 0.039 & 0.061 & 0.641 & 2.04 \\
\hline PsNAC92 vs. Pm025312 & 0.006 & 0.044 & 0.127 & 1.47 \\
\hline PsNAC93 vs. Pm020580 & 0.009 & 0.064 & 0.141 & 2.14 \\
\hline PsNAC94 vs. Pm020961 & 0.004 & 0.005 & 0.828 & 0.18 \\
\hline PsNAC95 vs. $P m 021768$ & 0.012 & 0.040 & 0.295 & 1.34 \\
\hline PsNAC95 vs. Pm021749 & 0.007 & 0.017 & 0.437 & 0.56 \\
\hline PsNAC96 vs. Pm021945 & 0.072 & 0.073 & 0.982 & 2.44 \\
\hline PsNAC99 vs. Pm022529 & 0.007 & 0.010 & 0.734 & 0.32 \\
\hline PsNAC102 vs. $P m 022820$ & 0.003 & 0.004 & 0.847 & 0.13 \\
\hline
\end{tabular}

${ }^{\mathrm{z}}$ Million years ago. 
Supplemental Table 8. The Pearson correlation value, linear fitting equation, and R squared $\left(\mathrm{R}^{2}\right)$ values between RNA-sequencing (RNASeq) data and quantitative real-time polymerase chain reaction (qRT-PCR) of 15 PsNACs.

\begin{tabular}{|c|c|c|c|}
\hline Gene & Pearson correlation value & Linear fitting equation & $\mathrm{R}^{2}$ \\
\hline$\overline{P S N A C 9}$ & $0.885^{*}$ & $\mathrm{y}=0.07 \mathrm{x}+0.07$ & 0.0783 \\
\hline PsNAC59 & $0.996^{* *}$ & $y=0.06 x+0.12$ & 0.992 \\
\hline PsNAC6 & $0.999 * *$ & $\mathrm{y}=0.09 \mathrm{x}+0.21$ & 0.997 \\
\hline PsNAC33 & $0.926^{* *}$ & $y=0.06 x+0.02$ & 0.857 \\
\hline PsNAC51 & $0.999 * *$ & $y=0.02 x+0.07$ & 0.999 \\
\hline PsNAC67 & $0.995 * *$ & $y=2.82 x+4.88$ & 0.989 \\
\hline PsNAC4 & $0.929 * *$ & $\mathrm{y}=0.01 \mathrm{x}+0.16$ & 0.863 \\
\hline PsNAC37 & $0.947 * *$ & $y=0.04 x+0.32$ & 0.0897 \\
\hline PsNAC13 & $0.997 * *$ & $\mathrm{y}=0.03 \mathrm{x}+1.43$ & 0.994 \\
\hline PsNAC50 & $0.904^{*}$ & $y=9.78 E-03 x+0.27$ & 0.817 \\
\hline PsNAC64 & $0.991 * *$ & $y=0.04 x+0.01$ & 0.981 \\
\hline PsNAC10 & $0.920 * *$ & $y=0.07 x+0.03$ & 0.847 \\
\hline PsNAC94 & $0.966 * *$ & $\mathrm{y}=0.04 \mathrm{x}+0.09$ & 0.933 \\
\hline PsNAC51 & $0.920 * *$ & $y=2.09 E-03 x+0.48$ & 0.847 \\
\hline PsNAC32 & 0.781 & $y=0.06 x+0.11$ & 0.61 \\
\hline
\end{tabular}

** and * represented a significant correlation at 0.01 level and 0.05 level (two-tailed), respectively.

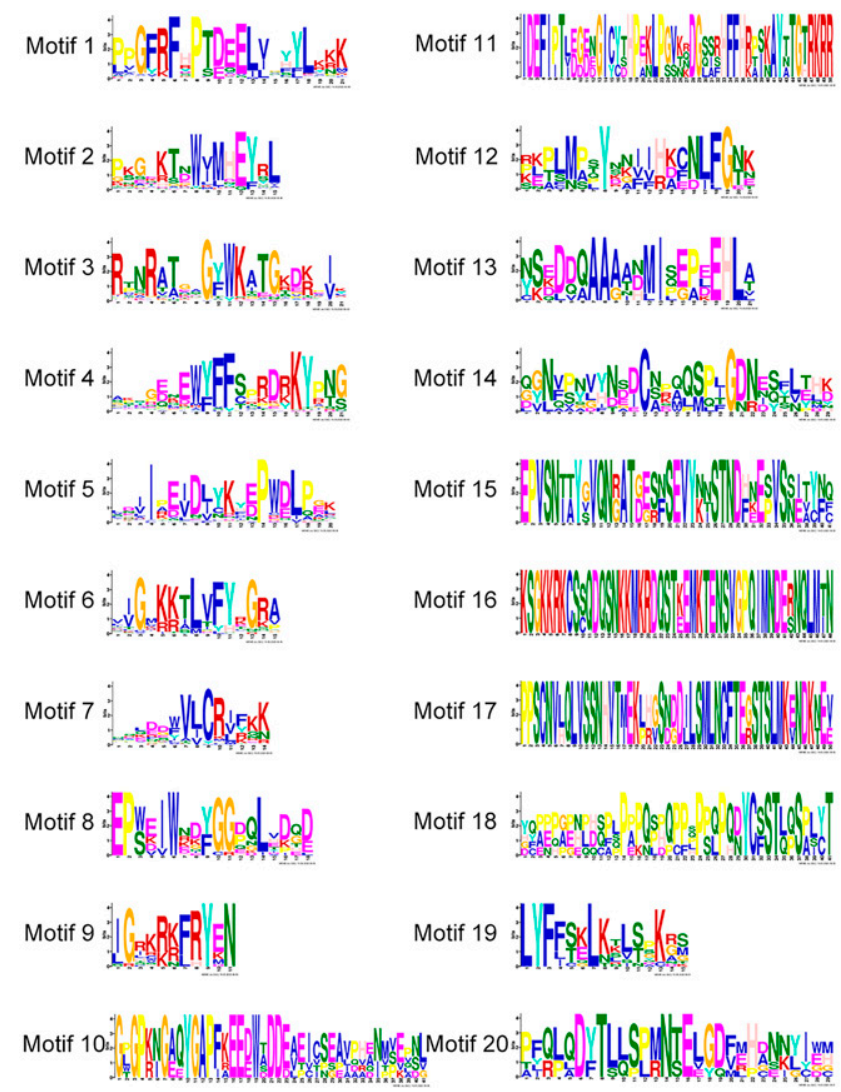

Supplemental Fig. 1. Sequence information for 20 conserved motifs of PsNACs. 
A

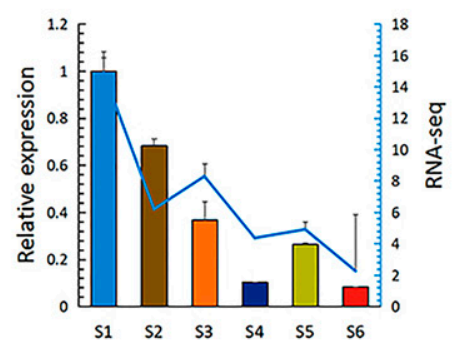

D

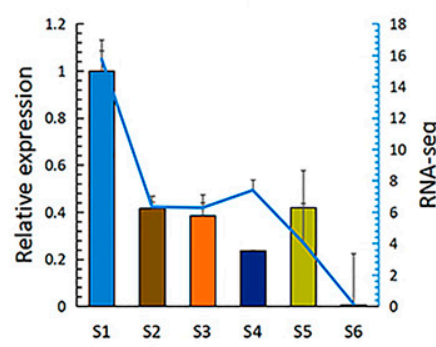

G

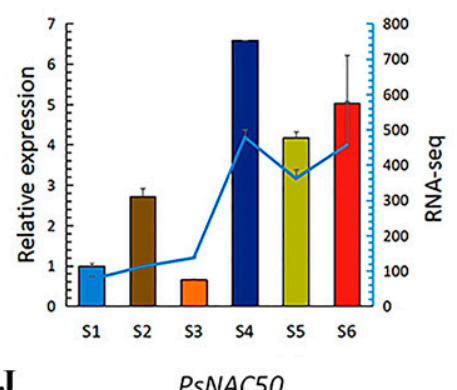

J

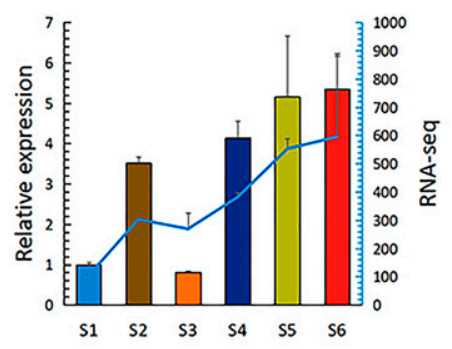

M

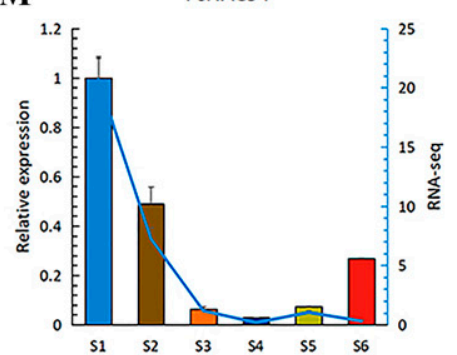

B

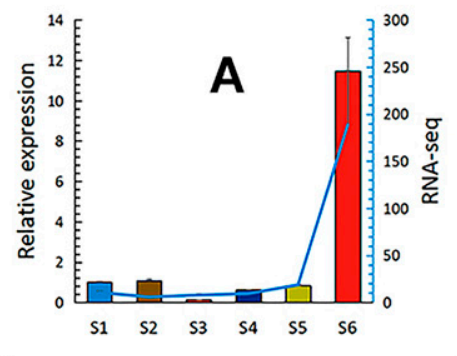

E

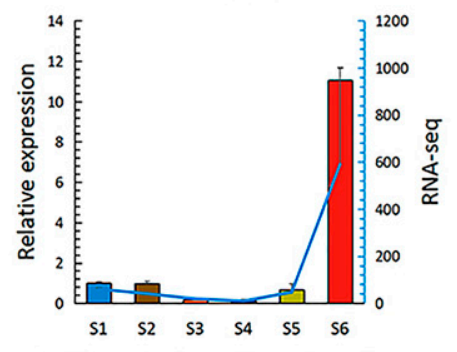

H

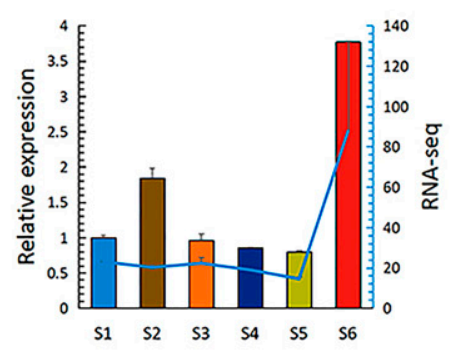

K

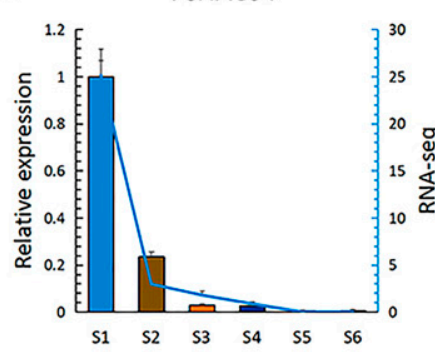

N

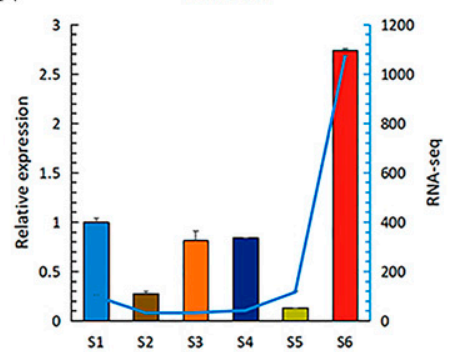

C

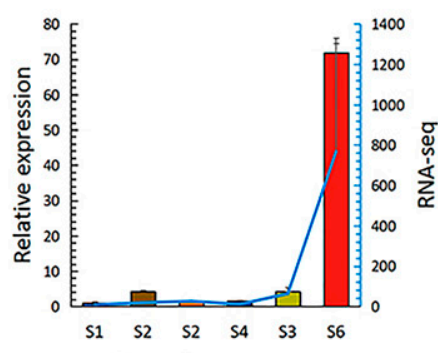

F

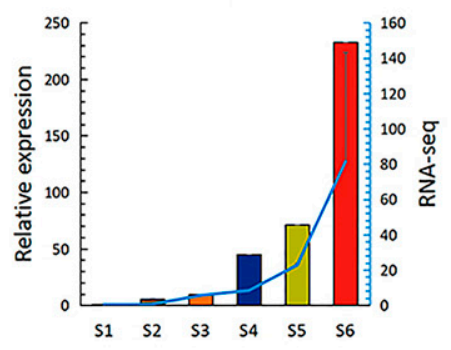

I

PsNAC13

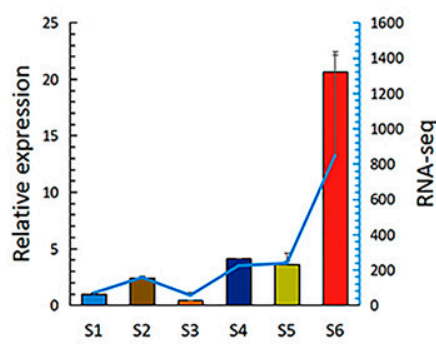

L

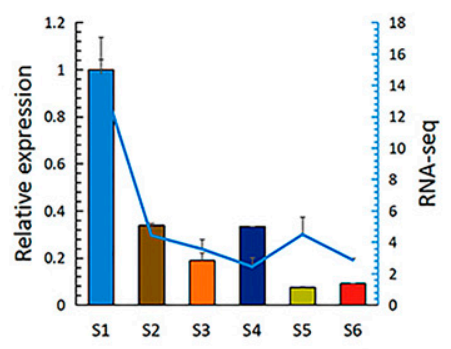

O

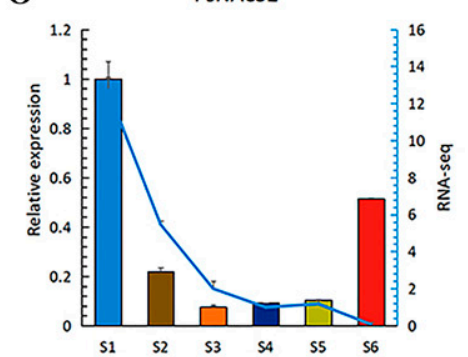

Supplemental Fig. 2. Expression analysis of $P s N A C s$ during siberian apricot fruit and kernel development by RNA-sequencing (RNA-Seq) data and quantitative real-time polymerase chain reaction (qRT-PCR). (A-K) PSNACs of fruit development; and (L-O) PsNACs of kernel development. Data were normalized to Ubiquitin-conjugating enzyme $(U B C)$ gene and vertical bars indicate standard deviation. QRT-PCR value were given as means \pm SD of three independent biological replicates. Column and line in the chart represented qRT-PCR and RNA-seq, respectively. 